\title{
SiO Maser Emission and the Intrinsic Properties of Mira Variables
}

\author{
Nimesh A. Patel ${ }^{1,2}$, Antony Joseph \& R. Ganesan Raman Research Institute, \\ Bangalore 560080
}

Received 1992 June 15; accepted 1992 August 17

\begin{abstract}
This paper presents observations of $\mathrm{SiO}$ maser emission from 161 Mira variables distributed over a wide range of intrinsic parameters like spectral type, bolometric magnitude and amplitude of pulsation. The observations were made at $86.243 \mathrm{GHz}$, using the $10.4 \mathrm{~m}$ millimeter-wave telescope of the Raman Research Institute at Bangalore, India. These are the first observations made using this telescope. From these observations, we have established that the maser emission is restricted to Miras having mean spectral types between M6 and M10. The infrared period-luminosity relation for Mira variables is used to calculate their distances and hence estimate their maser luminosities from the observed fluxes. The maser luminosity is found to be correlated with the bolometric magnitude of the Mira variable. On an $\mathrm{H}-\mathrm{R}$ diagram, the masing Mira variables are shown to lie in a region distinct from that for the non-masing ones.
\end{abstract}

Key words: stars-SiO masers-stars-long period variables-star-slate type-masers-SiO_radio lines-molecular

\section{Introduction}

It is well known for more than a decade now, that many oxygen-rich Mira variables exhibit the $\mathrm{SiO}$ maser phenomenon. This emission has been observed in rotational transitions of excited vibrational states upto $v=3$, (Alcolea, Bujarrabal \& Gallego 1989; Jewell et al. 1985). One of the basic questions that has remained poorly investigated is that of the relationship between the maser emission and the intrinsic properties of the Mira variables. This question has a bearing on the understanding of both. So far, about 190 late-type stars have been observed by millimeter wave telescopes, and about 40 per cent of them have shown maser emission (Engels \& Heske 1989). Preliminary attempts at studying the correlation of the maser power with spectral type were made by Cahn (1977) and Spencer et al. (1977).

Cahn (1977) reported a correlation between the absolute maser luminosity and the spectral type at maximum light and concluded that a Mira variable of any mean spectral type attains maximum value of maser power on approaching the zero phase of pulsation corresponding to the maximum light. Then, knowing the spectral type

\footnotetext{
${ }^{1}$ Also Joint Astronomy Programme, Indian Institute of Science Bangalore 560012.

2 Present Address: Five College Radio Astronomy Observatory, University of Massachusetts, Amherst 01033, Massachusetts, USA .
} 
and the pulsational phase of the star, one can predict its maser power, and use its measured value to obtain the distance to the Mira variable. The range of spectral types covered by Cahn was from M6 to M10, and 15 sources were considered in his study. No negative detections were included. Subsequently, Dickinson et al. (1978), found seven new Mira variables to be masing, and noted that all of them had spectral types later than M4. Two of these, i.e. Y Cas and RT Aql, were observed at near zero phase and allowed easy confrontation with the above mentioned correlation. While Y Cas fitted with the correlation, RT Aql did not. The dependence of maser luminosity on spectral type earlier than M6, not considered by Cahn, remained unanswered due to lack of adequate observations.

Spencer et al. (1977) noted that at spectral types near M8, the probability for a Mira variable to show $\mathrm{SiO}$ maser emission is greater than 40 per cent. Their sample consisted of 81 stars with very few objects earlier than M6. Their detection limit was $30 \mathrm{Jy}$ and their conclusions were based on fluxes, without taking distances into account.

In this paper, we report observations of 161 (mostly Mira) variables made at 86.243 GHz corresponding to the $\mathrm{J}: 2 \rightarrow 1, v=1$ transition in $\mathrm{SiO}$. One of the motivations of these observations was to study the dependence of the $\mathrm{SiO}$ maser luminosity on intrinsic properties of Mira variables. Therefore, we selected the sources to cover a wide range in spectral type. These observations are the first ones made using the Raman Research Institute (RRI) $10.4 \mathrm{~m}$ telescope at Bangalore, India. The method of observation and the instrumental characteristics are briefly described in section 2, (for more details see Patel 1990). In section 3, we present the list of sources we have observed, and the results of our observations. The integrated fluxes are converted to luminosities in section 4 . The correlations between the maser luminosity and the intrinsic properties of Mira variables, that have emerged from our observations, are described in section 5. Finally, in section 6, we discuss these results and attempt their interpretation.

\section{Observations and results}

\subsection{Observations using the RRI 10.4 m Telescope}

The $10.4 \mathrm{~m}$ millimeter-wave telescope at the Raman Research Institute in Bangalore, is a Cassegrain type antenna on an altitude-azimuth mount, with the receiver at the Nasmyth focus. This telescope is located on campus, at a latitude $=13^{\circ} 00^{\prime} 44.46^{\prime \prime}$, longitude $=77^{\circ} 34^{\prime}$ 59.67", and at an elevation of 930 meters above mean sea level. The front-end receiver is a wave-guide mounted Schottky-barrier diode mixer followed by an IF Amplifier at $1.4 \mathrm{GHz}$, both cooled to about $20 \mathrm{~K}$. The radiation is coupled by a lens to a quasi-optical diplexer which is closely attached to the dewar containing the mixer and the IF amplifier. The local oscillator is a Gunn-oscillator tunable from 85 to $120 \mathrm{GHz}$. The back-end used for the $\mathrm{SiO}$ observations consisted of a synchronousdetector for continuum measurements (for pointing and gain calibrations) and the following spectrometers: a 256 channel filter-bank having a resolution of $250 \mathrm{KHz}$ $(0.87 \mathrm{~km} / \mathrm{s}$ at $86 \mathrm{GHz})$, and an acousto-optical spectrometer having an effective resolution of $210 \mathrm{KHz}(0.71 \mathrm{~km} / \mathrm{s}$ at $86 \mathrm{GHz})$, over a total bandwidth of $120 \mathrm{MHz}$.

The observations reported here were made during three seasons in 1988, 1989 and 1990. The typical values of double side-band system temperatures at zenith were 
between 400 and $500 \mathrm{~K}$. The observations were calibrated using a chopper-wheel positioned between the diplexer and the lens. The zenith optical depth $\tau_{0}(\mathrm{z})$, frequently monitored by taking dip scans on the sky, had typical values between 0.1 and 0.5 . The r.m.s. pointing error was about 5 arcsec, as found from continuum scans of Jupiter and 5-point grids made on strong $\mathrm{SiO}$ sources. The aperture efficiency $\eta_{A}$, was calculated from continuum scans taken on Jupiter and Venus. The mean value of $\eta_{A}$ was 0.45 , during 1988 and 1989; for the 1990 data, $\eta_{A}$ was found to be a function of elevation, due to a possible misalignment in the feed-horn inside the dewar. The value of $\eta_{A}$ during this season was between 0.3 and $0 \cdot 4$. The measured fluxes of sources observed during this season, have been corrected for this effect. Furthermore, all observations made between IST $12 \mathrm{hrs}$ to $18 \mathrm{hrs}$ have been corrected for a drop in the gain of the telescope, due to the differential heating of the dish by the Sun. The reduction in the sensitivity of the telescope during daytime is about 20 per cent.

The variations in the detected power due to the inhomogeneous and varying atmosphere, and due to gain variations in the receiver, were cancelled by beam-switching which was achieved by tilting the tertiary mirror. The amount of beam thrown on the sky was about $80 \operatorname{arcsec}( \pm 40 \operatorname{arcsec}$ about the center of the beam).

Observations were made using a dual beam switching method. In this method two beam switched spectra are acquired, in which the respective reference positions straddle the source in azimuth. A residual ripple with a peak to peak antenna temperature of about 0.5 to $1 \mathrm{~K}$, which was present in each of these spectra, can be cancelled by averaging, leading to a very flat baseline as seen in Fig. 1.

\subsection{Selection of Sources}

The observed Mira variables were selected mostly from The General Catalogue of Variable Stars (Kholopov 1985, henceforth GCVS) and in a few cases from Gezari, Schmitz \& Mead (1984). Since one of our aims was to check the hypothesis that $\mathrm{SiO}$ masers exist only in very late-type Mira variables (with mean spectral type greater than M6), we included a substantial number of objects at spectral types earlier than M6. We also attempted to select those objects which have been well studied at near infrared wavelengths, so that we can obtain information about the variation of spectral types during pulsation, and its relation to the maser emission. Thus, we had many objects which are common to the catalogue of Lockwood (1972). To avoid very long integrations, an attempt was made to exclude objects that are at a distance greater than $1 \mathrm{kpc}$ from the Sun, by restricting the visual magnitude to lie below 15 magnitudes.

The list of selected objects along with their intrinsic properties is given in Table 1 in cols. 2-7. Among these, period was available for 143 objects. Fig. 2 shows their distribution in period. For 28 sources the spectral type was either unknown or not M. The distribution of the remaining 133 sources in the mean spectral type is shown in Fig. 3. The similarity of these histograms with the corresponding ones for the galactic Mira variables (Ikaunieks 1975), shows that the sample we have selected is unbiased.

The phases of pulsation $(\phi)$ and the visual amplitude of pulsation $\left(\Delta m_{v}\right)$ for some of our sources were provided by the American Association of Variable Star Observers (AAVSO). These occur in cols. 8 and 17 of Table 1. 


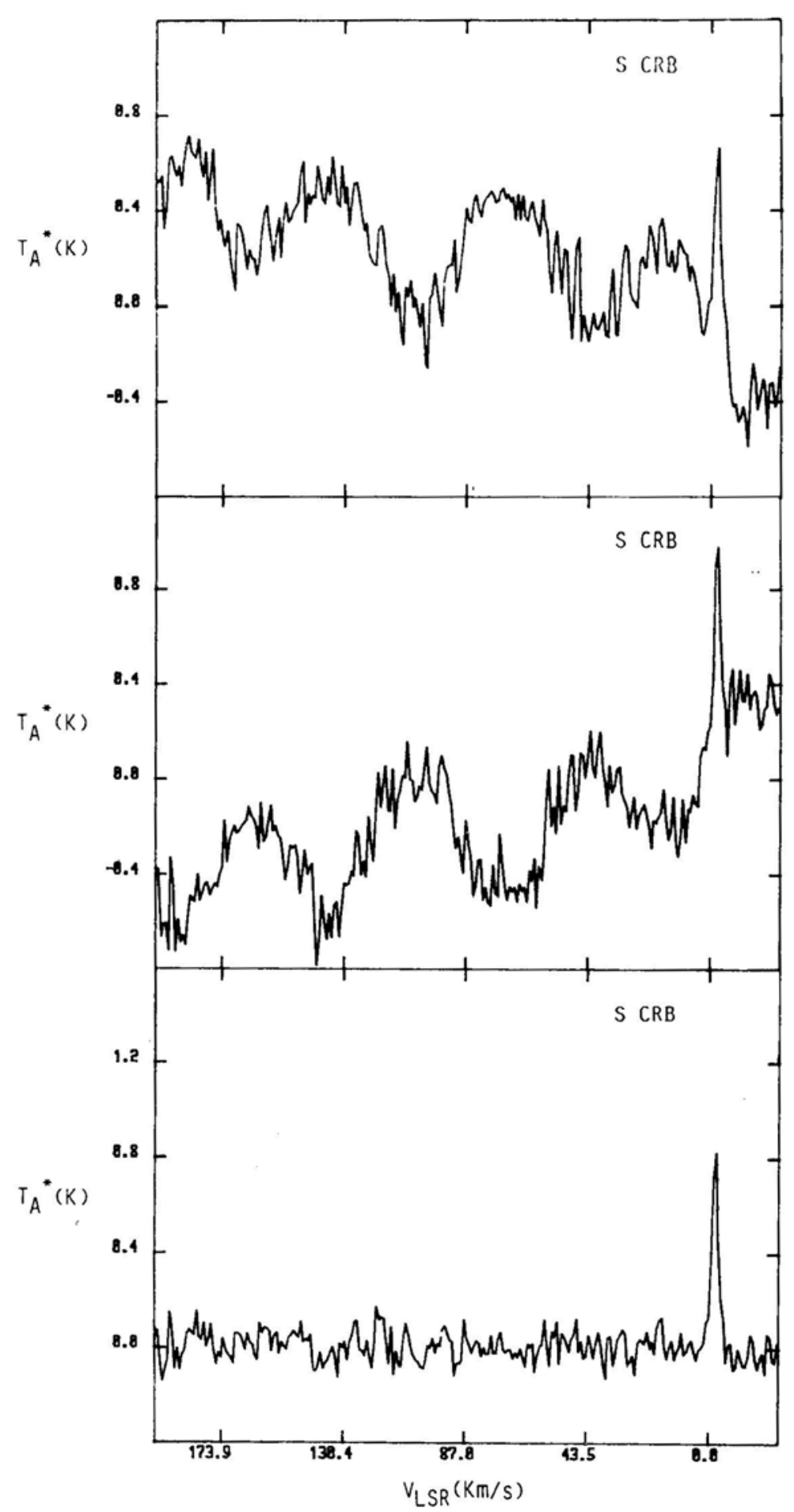

Figure 1. Beam-switched spectra acquired with offsets in azimuth, to one side (top panel), to the opposite side (middle panel) and their average (bottom panel). 


\begin{tabular}{|c|c|c|c|c|c|c|c|c|}
\hline \multirow{2}{*}{ 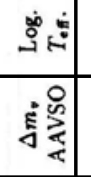 } & \multirow{2}{*}{ 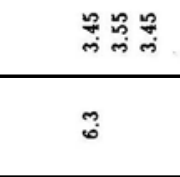 } & 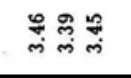 & \multirow[t]{2}{*}{ 俩 } & \multicolumn{2}{|c|}{ 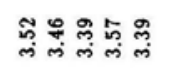 } & \multirow{2}{*}{ 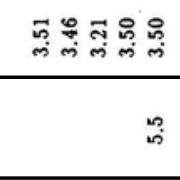 } & 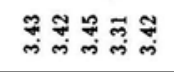 & \multirow{2}{*}{ 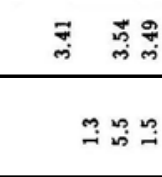 } \\
\hline & & $\stackrel{\infty}{\infty} \stackrel{0}{\circ}$ & & i. $\stackrel{\circ}{-}$ & is & & ?ำ & \\
\hline 焉竞 & & 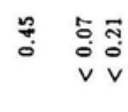 & $\begin{array}{l}\overrightarrow{0} \\
\dot{0} \\
v\end{array}$ & 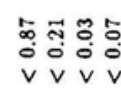 & 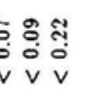 & 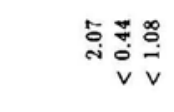 & 灾 & 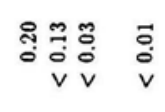 \\
\hline 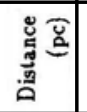 & & 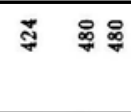 & $\bar{\Xi}$ & 덤 : : & $8 \%$ & 吕总总 & 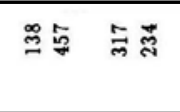 & 㔛䨐斯 œ \\
\hline$\Xi_{0}^{\Xi}$ & & $\stackrel{\infty}{\circ}$ & & & & $\stackrel{\circ}{\circ}$ & 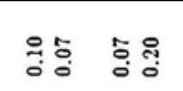 & : \\
\hline 0 & 궁웅형영 웅 & 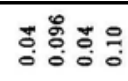 & 苂 & 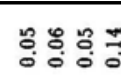 & t: & 웅웅웅웅웅 & 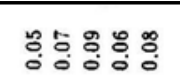 & 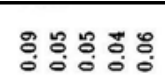 \\
\hline$\vec{\neg}$ & & $\stackrel{2}{*}$ & & & & $\stackrel{M}{i}$ & $\stackrel{0}{0} \underset{\infty}{\infty} \stackrel{n}{m}$ & $\stackrel{\infty}{-}$ \\
\hline$>\stackrel{\infty}{\underline{E}}$ & & $\stackrel{\infty}{\substack{1 \\
1}}$ & & & & $\stackrel{n}{\infty}$ & 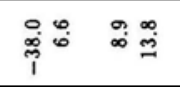 & $\stackrel{n}{p}$ \\
\hline$\therefore \square$ & & 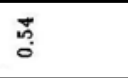 & & & & ल्ल. & 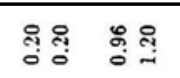 & ঞ্ণু \\
\hline 立 & 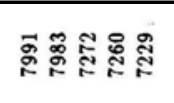 & 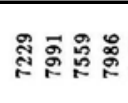 & 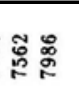 & 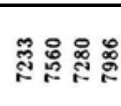 & 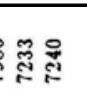 & 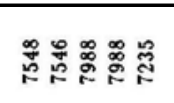 & 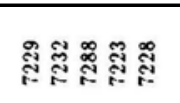 & 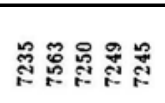 \\
\hline$a$ & $\begin{array}{ll}\vec{H} & \stackrel{0}{0} \\
0 & 0 \\
0\end{array}$ & 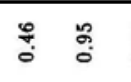 & $\stackrel{0}{0}$ & 영 훙: & $\underset{\infty}{\infty} \stackrel{\infty}{:}$ & 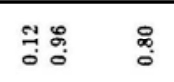 & 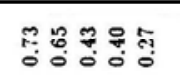 & m్ల్లో \\
\hline 量要 & ळ్ల ळ్ల & 矛㗊馬 & $\$$ & 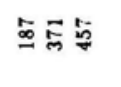 & 串 & 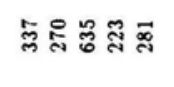 & 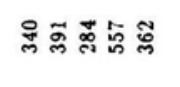 & జ \\
\hline 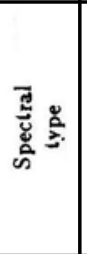 & 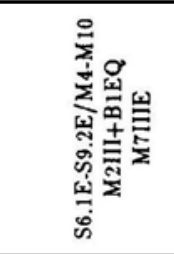 & 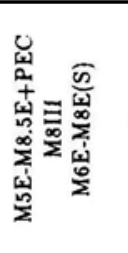 & 莣莣 & 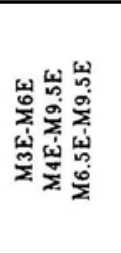 & 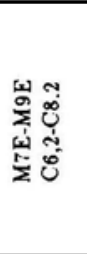 & 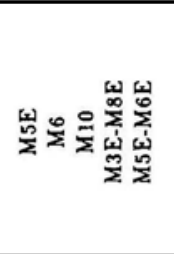 & 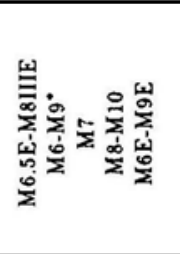 & 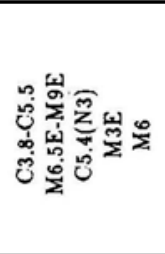 \\
\hline 竞 & $\sum \sum_{N} z$ & 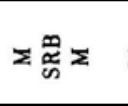 & $\Sigma \frac{\infty}{4}$ & $\Sigma \Sigma \Sigma$ & $\Sigma \Sigma$ & $\Sigma \Sigma \Sigma \Sigma \Sigma$ & 胥 $\Sigma \Sigma \Sigma \Sigma$ & 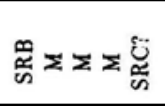 \\
\hline 熍 & 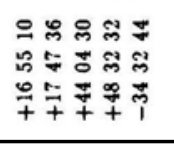 & 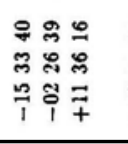 & 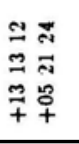 & 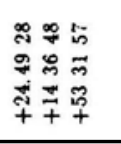 & 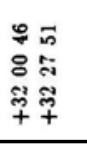 & 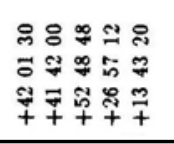 & 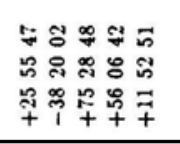 & 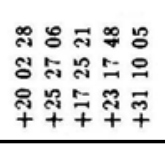 \\
\hline ڤั & 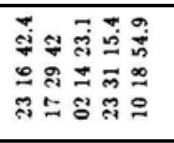 & 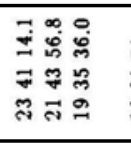 & 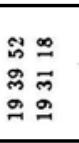 & 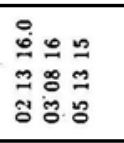 & 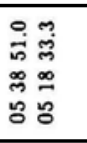 & 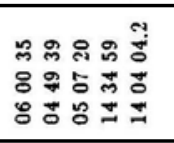 & 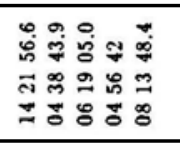 & 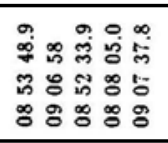 \\
\hline ֻั̆ & 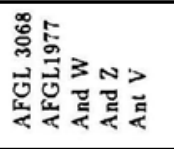 & 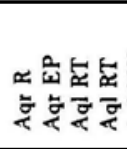 & 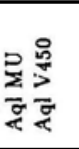 & 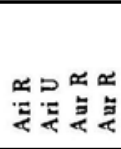 & 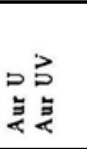 & 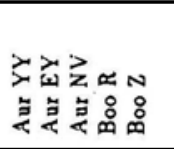 & 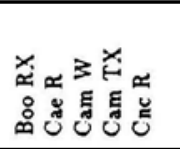 & 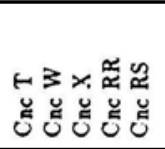 \\
\hline$\dot{0}$ & - बam w in & $\infty \cdots \infty$ & 옹 & 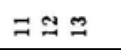 & 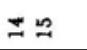 & 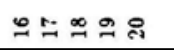 & 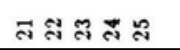 & 유소 స̊요 \\
\hline
\end{tabular}




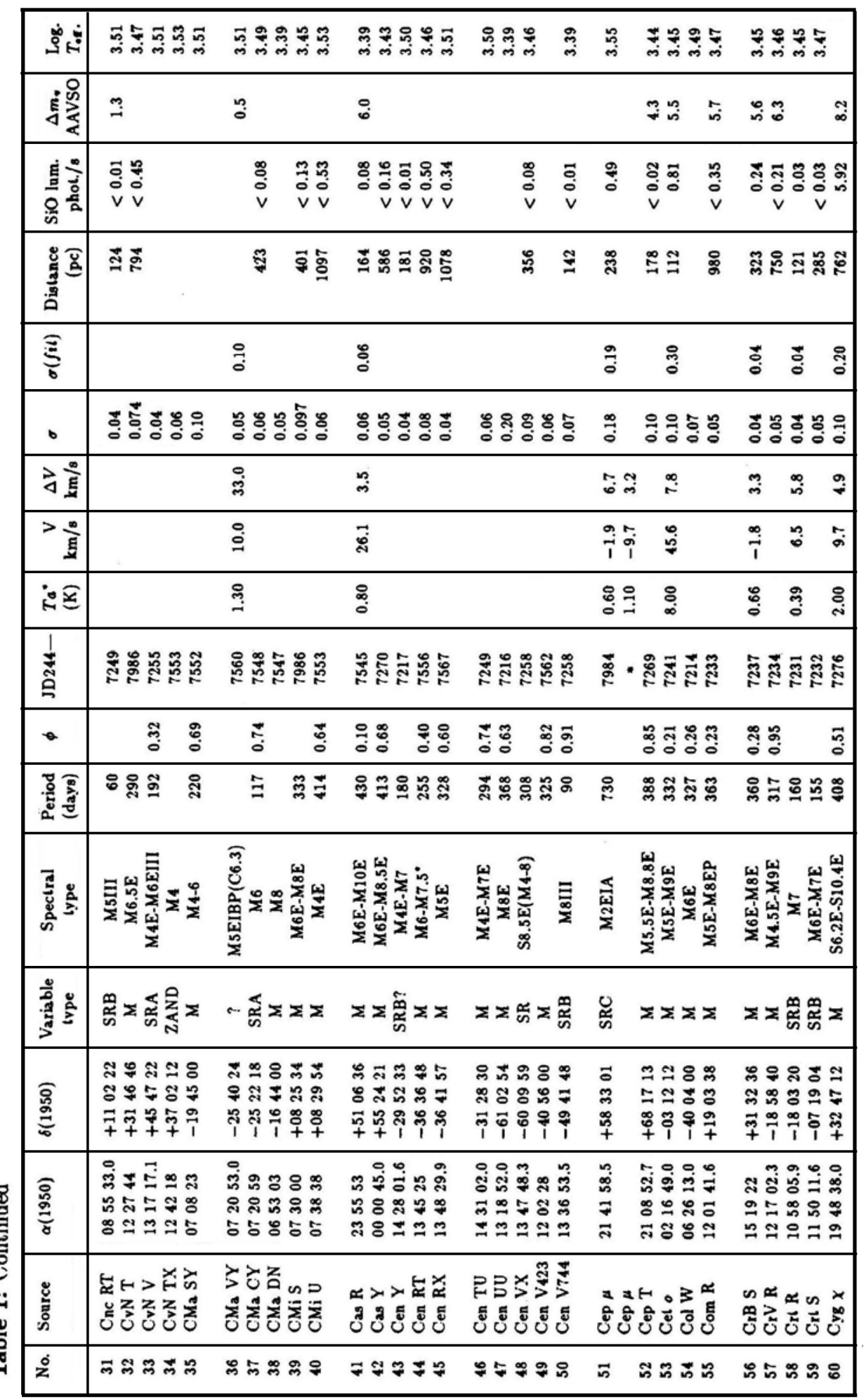




\begin{tabular}{|c|c|c|c|c|c|c|c|}
\hline 今ึ่ & 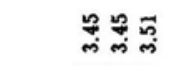 & 疍 & 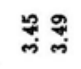 & 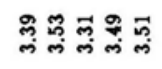 & 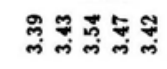 & 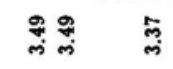 & F \\
\hline 歼总 & 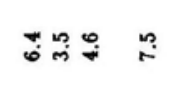 & & & กี & 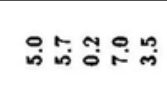 & 車 & $\stackrel{ }{-} \underset{i}{\circ}$ \\
\hline 豆总 & 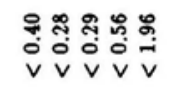 & $\stackrel{0}{\circ}$ & 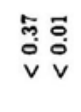 & 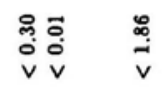 & 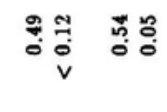 & 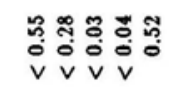 & 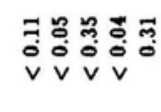 \\
\hline 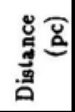 & స్థ & $:$ & $\tilde{\Xi}$ & 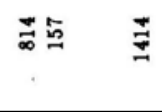 & 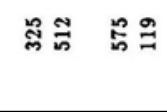 & ర్ల్రం & 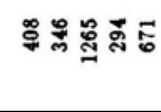 \\
\hline छั & & స్్ & & & $\stackrel{\infty}{\circ}$ & श̊. & : \\
\hline 0 & 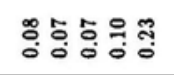 & 눙 & : & 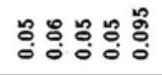 & 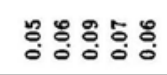 & 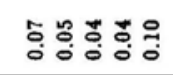 & : \\
\hline$\vec{\triangleleft}$ & & in & & & స్ల $\quad \stackrel{\infty}{n}$ & $\ddot{m}$ & $\stackrel{\circ}{\circ}$ \\
\hline$>\frac{\infty}{\mathrm{g}}$ & & 웅 & & & $\stackrel{\vec{m}}{\vec{i}} \stackrel{\infty}{\dot{p}}$ & ले & $\stackrel{\circ}{i}$ \\
\hline$\therefore \widehat{2}$ & & $\circ$ & & & 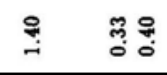 & ల్ల్ & ల్ల్ర \\
\hline$\frac{1}{ \pm}$ & 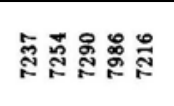 & 怘递 & . & 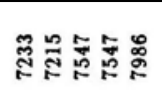 & 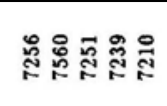 & 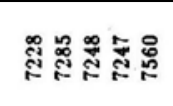 & 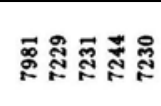 \\
\hline 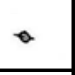 & 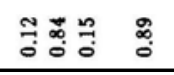 & $\begin{array}{l}\infty \\
\substack{\infty \\
0} \\
0\end{array}$ & & 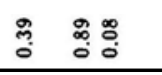 & 它 & 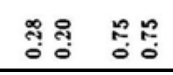 & ๕ั \\
\hline 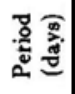 & 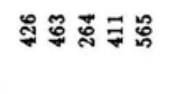 & $\stackrel{?}{*} \tilde{e}^{\infty}$ & 융 & 텅 \& & ஓ & 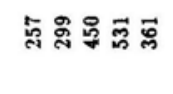 & 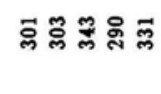 \\
\hline 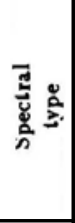 & 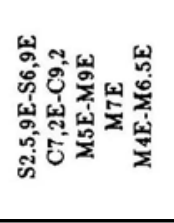 & 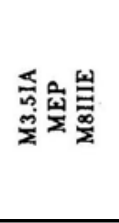 & 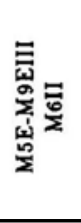 & 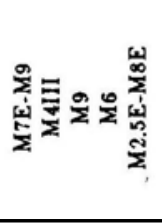 & 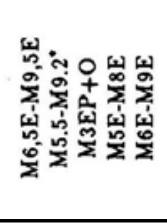 & 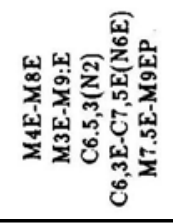 & 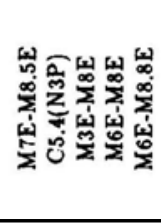 \\
\hline 造 & $\sum \Sigma \Sigma \Sigma \Sigma$ & 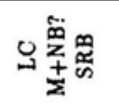 & $\Sigma \frac{\mathscr{E}}{\tilde{w}}$ & 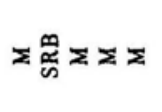 & 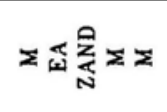 & 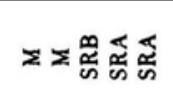 & $\Sigma \Sigma \Sigma$ 药 $\Sigma$ \\
\hline 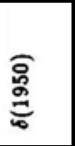 & 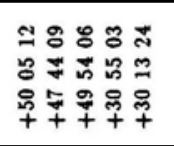 & 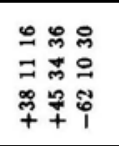 & 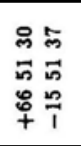 & 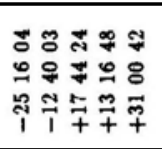 & 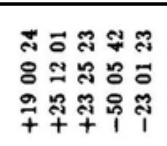 & 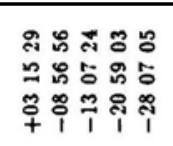 & 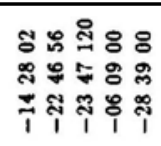 \\
\hline ฮ્ळ & 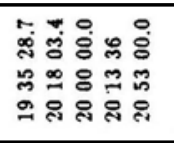 & 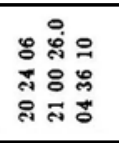 & 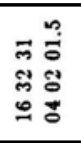 & 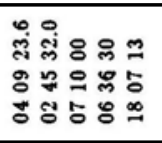 & 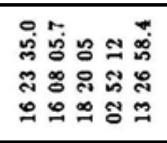 & 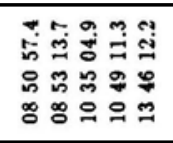 & 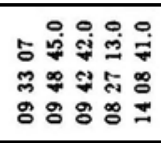 \\
\hline ๕ั้ & 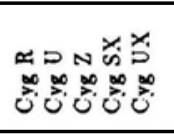 & 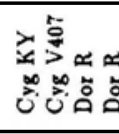 & 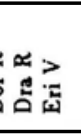 & 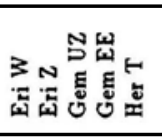 & 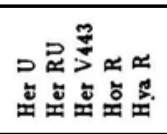 & 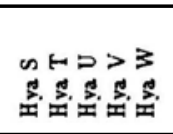 & 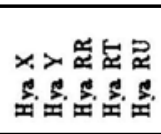 \\
\hline ì & ๑ & $\because 5 \%$ & $8:$ & 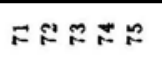 & 유: & $\vec{\infty} \underbrace{\infty}_{\infty} \infty$ & ○ \\
\hline
\end{tabular}




\begin{tabular}{|c|c|c|c|c|c|c|c|c|c|c|}
\hline نَّف & 苛 & & 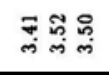 & $\underset{i}{F}$ & ल्ले & 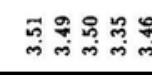 & & $\stackrel{?}{p}$ & i़ i़ क्ष & 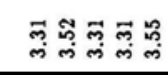 \\
\hline 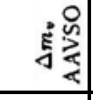 & & & $\underset{\sim}{\sim} \underset{\sim}{\infty}$ & 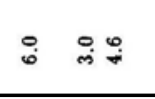 & & $\stackrel{\circ}{\text { is }}$ & $\stackrel{\circ}{+}$ & $\overrightarrow{i n}$ & $\stackrel{m}{9}$ & \\
\hline 혈 & & & $\stackrel{\text { åd }}{\stackrel{\infty}{+\infty}}$ & 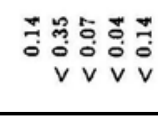 & & 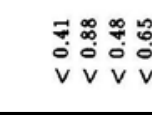 & & & 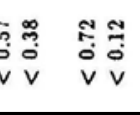 & 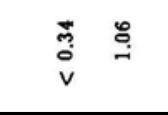 \\
\hline 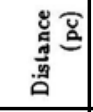 & & & ๑ ळ్ల్ల & స్సా & & 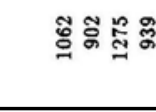 & & & $: \stackrel{9}{0}$ : & $\underset{\infty}{\infty}$ \\
\hline 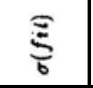 & 웅 & o̊ & $\stackrel{ }{0}$ & $\stackrel{\circ}{0}$ & & & & & & b. \\
\hline$\circ$ & 웅영융융 & م: & 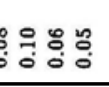 & 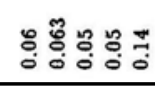 & & 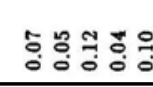 & & & 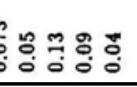 & 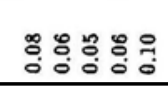 \\
\hline বे & $5:$ & $\stackrel{\circ}{-}$ & ल્ & $\stackrel{\square}{\circ}$ & & & & & & 9 \\
\hline$>\underset{\Xi}{\Xi}$ & ஸึં & $\stackrel{\circ}{\dot{n}}$ & $\stackrel{\circ}{i}$ & $\stackrel{\leftrightarrow}{\longrightarrow}$ & & & & & & $\ddot{m}$ \\
\hline$\therefore \widehat{a}$ & †̛ & ت্் & 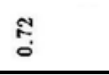 & ঙ্ণি & & & & & & జ్తి \\
\hline$\frac{1}{ \pm}$ & 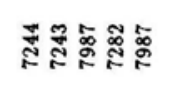 & $\vec{\infty} \underset{\infty}{\alpha}$ & 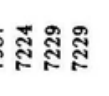 & 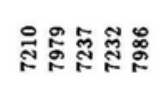 & & 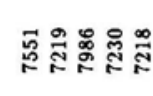 & & & 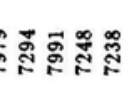 & 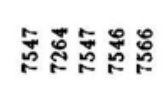 \\
\hline - & ฆั่ สี & & 눙 & : & : & 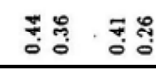 & & : & t. & 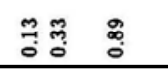 \\
\hline 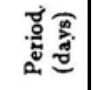 & 곡 & & 일 & 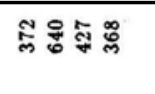 & 品 & 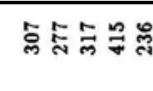 & & ల్ల & 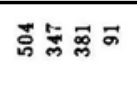 & శ్లి ఫ్ స్ \\
\hline बू. & 월 幽 & & 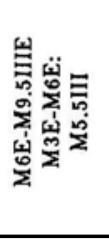 & 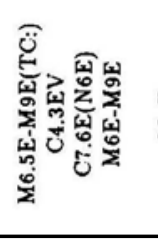 & 幽 & 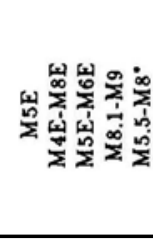 & & 幽 & 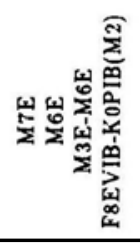 & 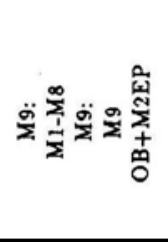 \\
\hline 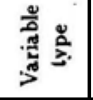 & $\Sigma \Sigma$ & & $\Sigma \Sigma \ddot{g}$ & 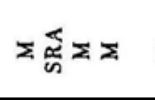 & $\Sigma$ & $\sum \Sigma \Sigma \Sigma \Sigma$ & & $\Sigma$ & 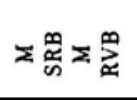 & 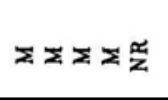 \\
\hline 高 & 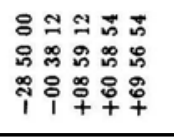 & 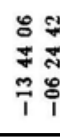 & 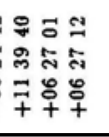 & 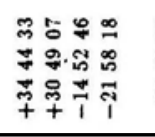 & 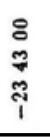 & 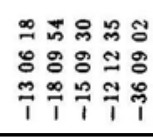 & & $\begin{array}{l}8 \\
\stackrel{8}{*} \\
\text { के } \\
+ \\
\end{array}$ & 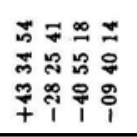 & 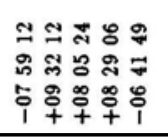 \\
\hline 용 & 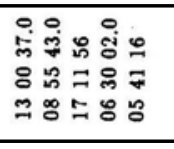 & 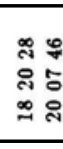 & 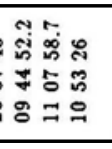 & 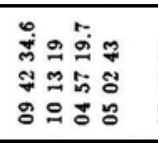 & \begin{tabular}{l}
$\infty$ \\
\multirow{2}{1}{} \\
0 \\
0 \\
0
\end{tabular} & 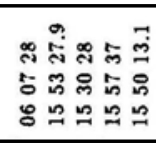 & & $\begin{array}{l} \\
0 \\
0 \\
\vdots \\
\vdots \\
\vdots \\
\end{array}$ & 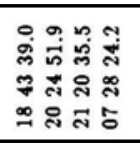 & 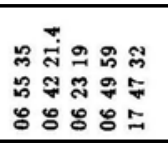 \\
\hline ๕ั้ & 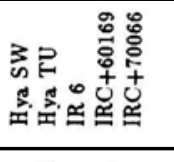 & 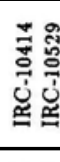 & 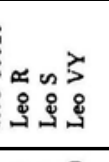 & 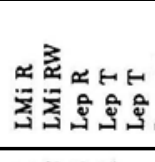 & & 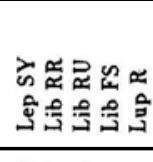 & & $\begin{array}{l}>> \\
3.5\end{array}$ & 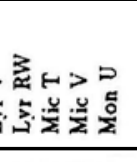 & 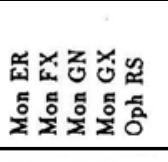 \\
\hline$\dot{z}$ & 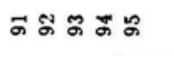 & \& 。 & ஃ̊ ஃ & 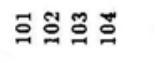 & $\stackrel{\circ}{\circ}$ & $\stackrel{\circ}{\circ} \stackrel{0}{\circ} \stackrel{\circ}{\circ}$ & & $\Xi$ & $\Xi \Xi \Xi \Xi$ & 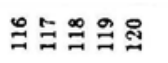 \\
\hline
\end{tabular}




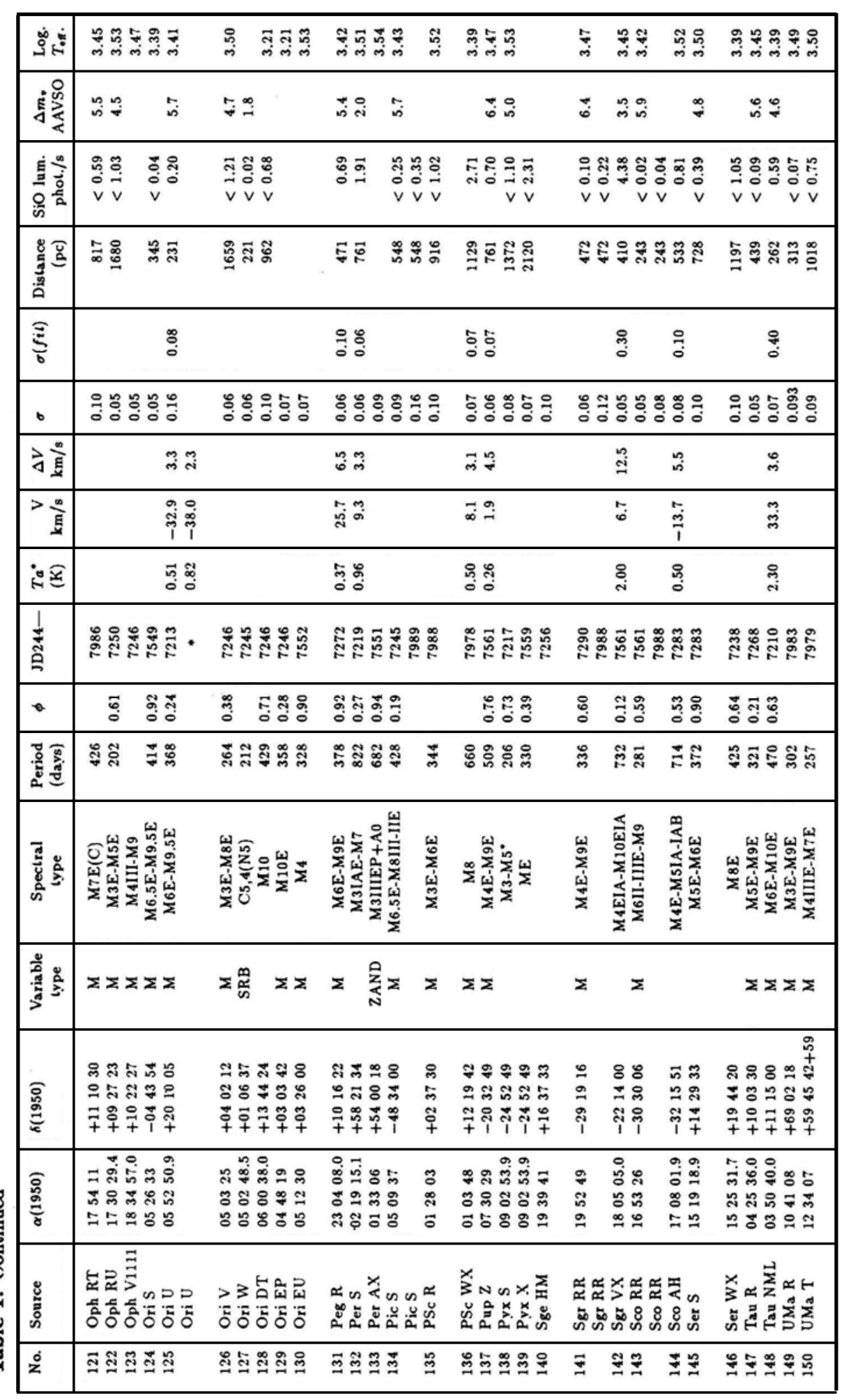




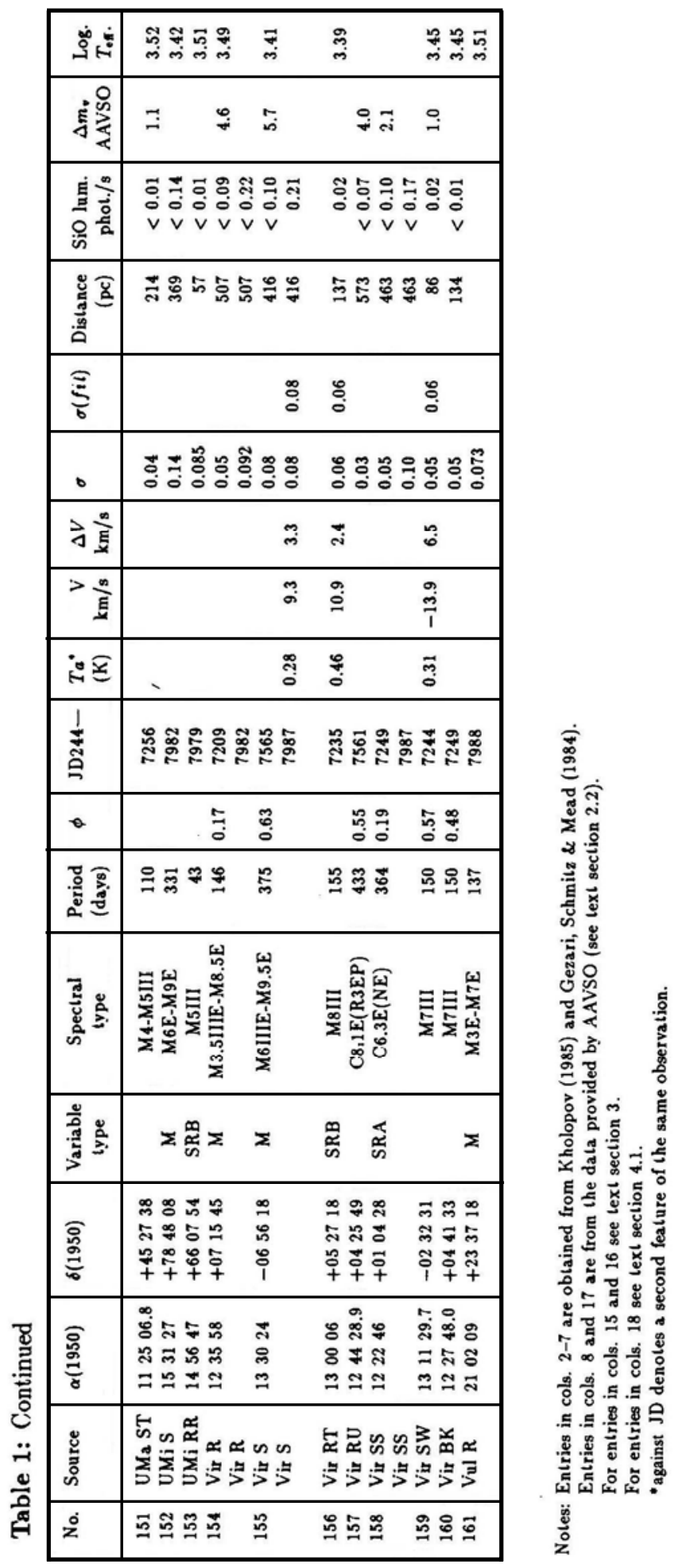




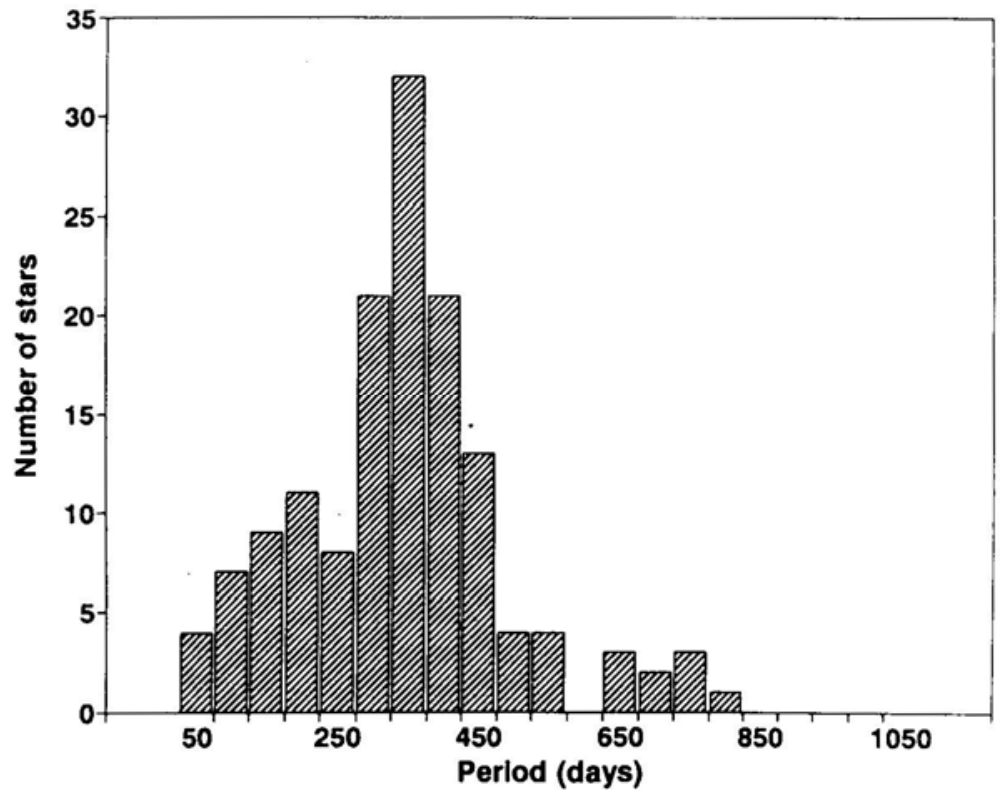

Figure 2. Distribution in pulsation period of the observed sources.

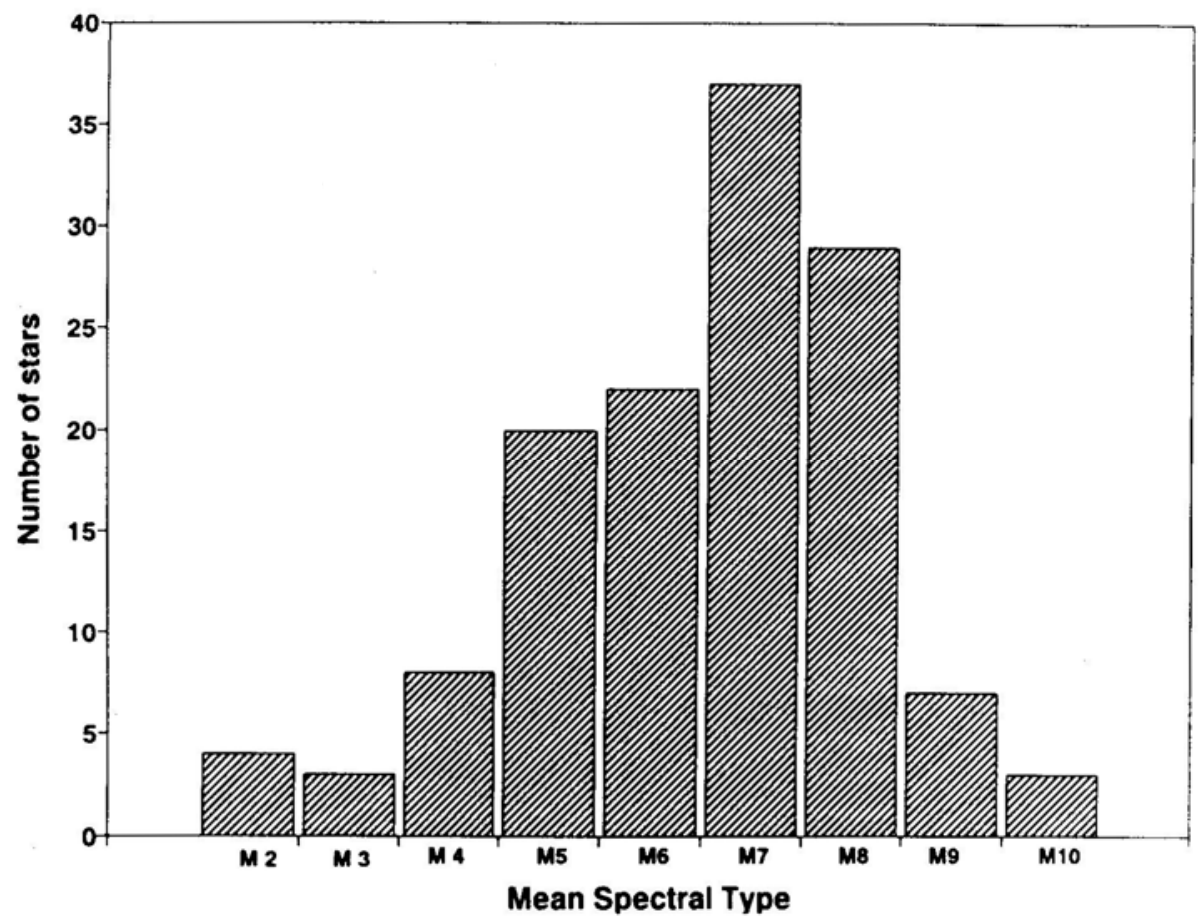

Figure 3. Distribution in mean spectral-types of the observed sources. 


\subsection{Results}

The results of our observations are presented in cols. 10-14 of Table 1. Of the 161 sources observed, 37 showed $\mathrm{SiO}$ maser emission. The fluxes ranged from 0.2 to $11 \mathrm{~K}$ (16 to $880 \mathrm{Jy}$ ) and the full width at half maximum from 1 to $10 \mathrm{~km} / \mathrm{s}$. The $1 \sigma$ noise level was 0.04 to $0.11 \mathrm{~K}$ (about 3 to $9 \mathrm{Jy}$ ).

Among the positive detections, the following seven sources are new detections at $86 \mathrm{GHz}$ not found in the compilation of Engels (1979), or Engels \& Heske (1989): $\mathrm{R}$ Cae, T Cnc, R Crt, R Hor, RU Hya, S Vir and SW Vir. Note that T Cnc is a C type star (Vardya 1989). In these stars the oxygen to carbon ratio is $<1$ and one expects $\mathrm{SiO}$ to be a less dominant Silicon species compared to say SiS (Sahai 1987); thus the presence of $\mathrm{SiO}$ maser emission in $\mathrm{T}$ Cnc is surprising (see also Lloyd Evans 1990). The spectra of some new detections, and of one negative detection baseline are shown in Fig. 4(a) \& (b). The parameters listed in cols. 9-14 of Table 1 are obtained by fitting gaussians to the line-profiles. In case of multiple peaks within the profile, we have fitted gaussians to the individual peaks, where we could discern the peaks to be separate. In blended features, we have not fitted more than one gaussian. To obtain upper limits on fluxes and luminosities for non-detections, we have used a mean value of $5 \mathrm{~km} / \mathrm{s}$ for the line-width, and $3 \sigma$, for an upper limit in antenna temperature, where $\sigma$ is the r.m.s. noise in the baseline.

\section{Distances of the Mira variables}

As a first step towards knowing the relationship between the maser phenomenon and the properties of the Mira variables, we convert the observed maser fluxes into luminosities.

To calculate the true maser luminosity, one needs to know or assume something about its isotropy. According to Alcock and Ross (1986), the maser emission is likely to be highly anisotropic. VLBI observations indicate emission from spots located around the star over a region of angular dimensions about 5 times the stellar radius (Moran et al. 1979; Lane 1984, McIntosh et al. 1989). It is likey that the emission is beamed and some of it is aimed towards us appearing as spots. However, at present there is not enough data (e.g., time-monitoring with VLBI), to conclude anything about the geometry of the total emission from the source. We can do no better than to assume that no matter from which direction one is looking at the Mira variable, one would see more-or-less the same fraction and that the filling factor is the same for all Miras. We have determined the distances to the Mira variables in our sample and obtained the maser luminosities from the observed integrated fluxes under the spectral lines by assuming isotropic emission.

Currently, the best available period-luminosity relation for Mira variables is in terms of the K-magnitudes, and is given by,

$$
M_{\mathrm{K}}=0.53-3.291 \log P
$$

with r.m.s. of about 0.1 magnitude (Feast 1984). We have used this relation to derive distances to Mira variables in our sample. Following Feast et al. (1982), we have corrected the apparent magnitudes for interstellar extinction, i.e.,

$$
A_{K}=\frac{0.12}{\sin b}\left(1-e^{-10 r \sin b}\right),
$$



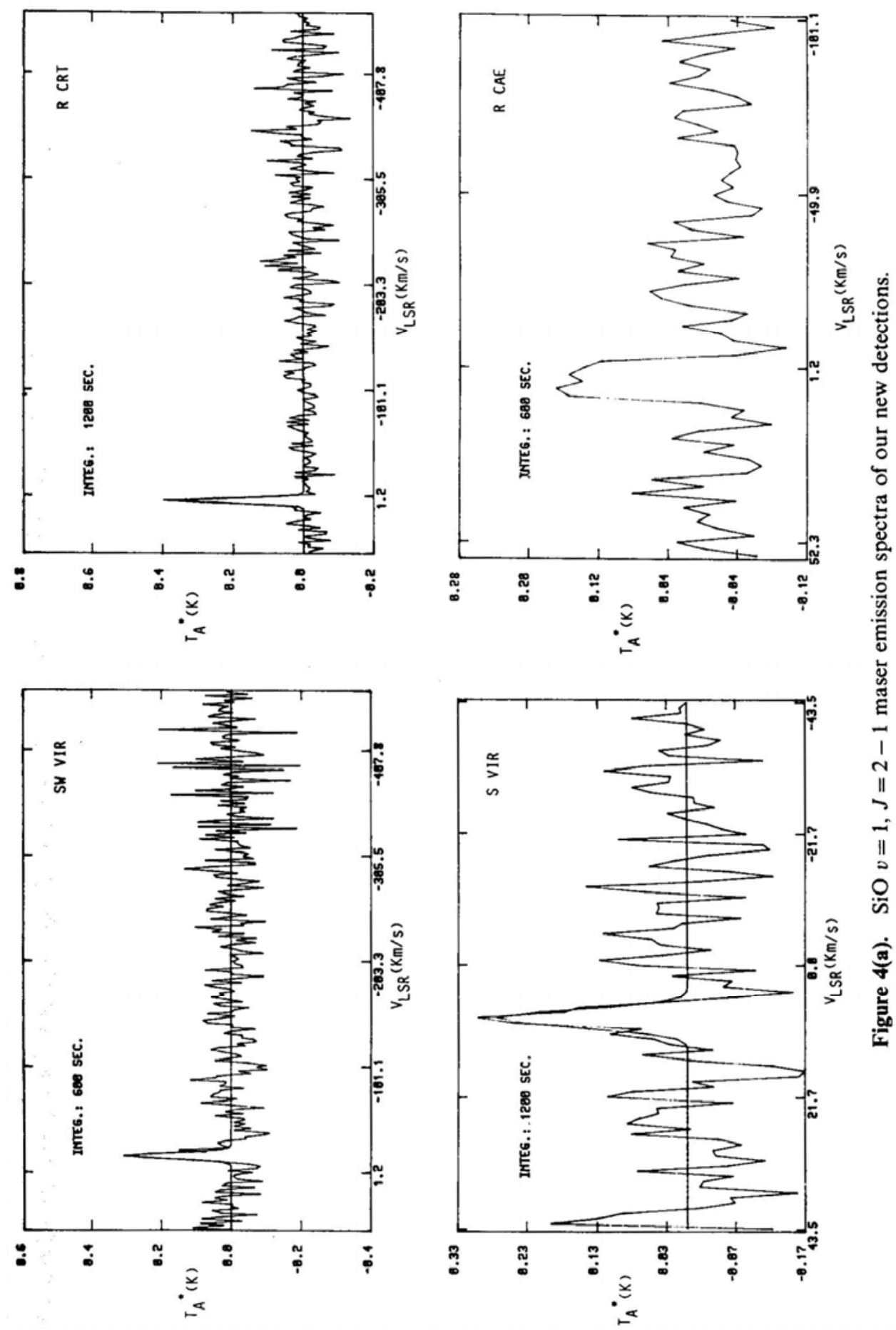

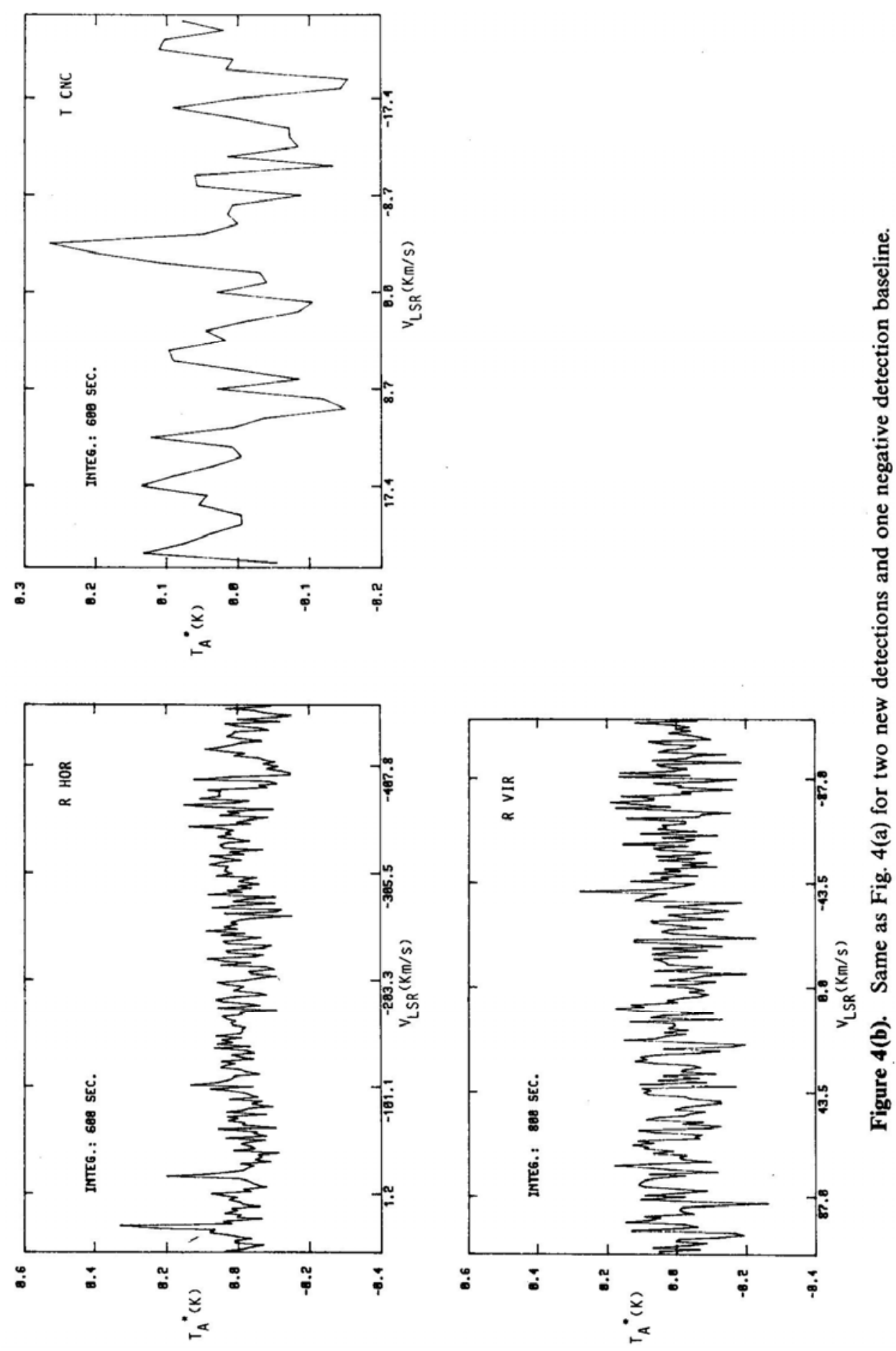


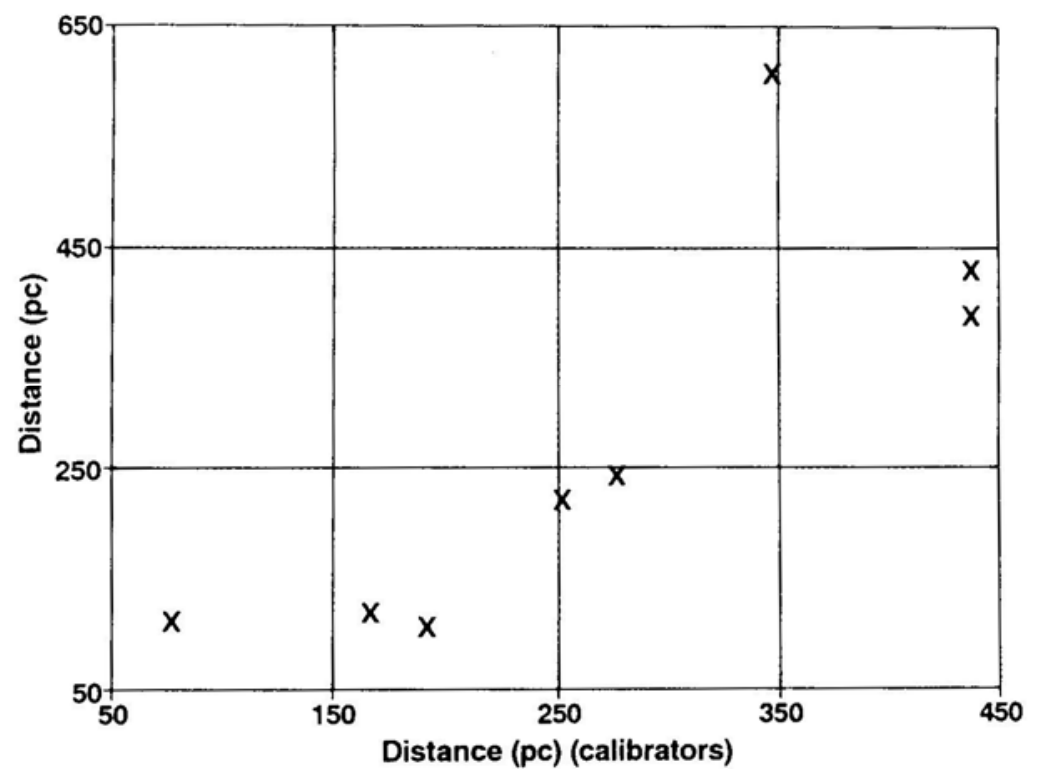

Figure 5. Comparison of our distances with the "Calibrator" distances (see text).

where $r$ is the distance in $\mathrm{kpc}$ and $b$ is the galactic latitude. The apparent $K$ magnitudes were obtained from Catchpole et al. (1979) and from Gezari, Schmitz \& Mead (1984). These derived distances, when plotted against the eight 'calibration' distances of Robertson \& Feast (1981) in Fig. 5, are in good agreement. IR distances are reliable due to their relative insensitivity to extinction corrections and we have therefore adopted them. These calculated distances and luminosities of the observed sources are listed in cols. 15 and 16 of Table 1.

A correlation between distances and some intrinsic stellar property is not expected unless there is a hidden bias in our distance determination method. A check confirmed no correlation.

\section{The SiO maser luminosity and intrinsic properties of Mira variables}

\subsection{Determination of Effective Temperature}

The spectral type of a Mira varies during its pulsation. The spectral types listed in column 6 of Table 1, are from the General Catalogue of Variable Stars (Kholopov 1985), and represent the extreme values during the pulsation cycles. The spectral types for several Mira variables at optical maximum are given by Keenan, Garrison \& Deutsch (1974), and throughout the pulsation period by Lockwood \& Wing (1971). The calibration of the spectral type with effective temperature for the Mira variable is full of uncertainties (Bessell et al. 1989).

Dyck, Lockwood \& Capps (1974) have obtained colour temperatures from black-body fits to their measured fluxes at 1.04 and 2.5 microns and have calibrated these colour temperatures with the effective temperatures of seven cool stars whose 


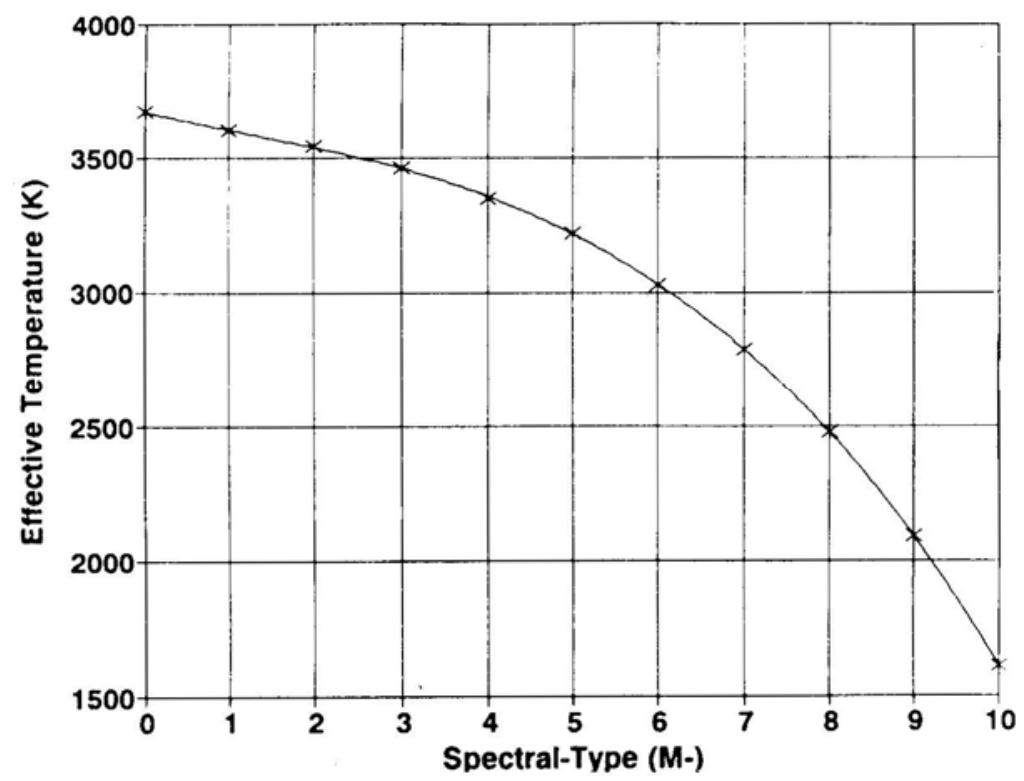

Figure 6. Dependence of effective temperature on the spectral-type of a Mira variable (from Dyck et al. 1974).

angular diameters have been measured. Their sample of stars contain Mira variables and IRC stars. The effective temperatures determined by this method, are shown in Fig. 6, which also shows a fitted polynomial. We have used this relation here. These temperatures appear in col. 18 of Table 1. One must note that the measured points are accurate to about half a division in spectral type. Since most of the energy is emitted at infrared wavelengths, it seems reasonable to adopt these temperatures which are obtained by measurements of fluxes and diameters at these wavelengths.

\subsection{The H-R Diagram for Mira Variables}

The absolute bolometric magnitudes from the P-L relation (Equation 1), and the effective temperatures obtained from spectral types as mentioned above, are used to plot an H-R diagram for the Mira variables in our sample. This is shown in Fig. 7. This figure may be compared with the H-R diagrams for Mira variables obtained previously by Keeley (1970), Scalo (1976) and Tsuji (1981). The H-R diagrams given by these authors are mainly for M-giant stars, including a very few Mira variables. As far as we know, Fig. 7 shows for the first time, the evolutionary status of the masing and non-masing Mira variables. From this we see that the masing sources lie in the region $\mathrm{M}_{\mathrm{bol}} \leqslant-4.8$ magnitude and $\log \left(\mathrm{T}_{\text {eff }}\right) \leqslant 3.47$. The four '\#' symbols indicate supergiants, i.e. S Per, AH Sco, $\mu$ Cep, and VX Sgr. The three SiO maser sources with $\mathrm{M}_{\mathrm{bol}} \cong-4.2$ are R Crt, RT Vir and SW Vir whose variable types are $\mathrm{SRB}$ or unknown (i.e. not Mira). The $\mathrm{SiO}$ luminosities of these are $\approx 0.03$ photons $s^{-1}$ which are about 5 per cent of the typical values observed for Mira variables. The cutoffs in $\mathrm{M}_{\mathrm{bol}}$ and $\mathrm{T}_{\text {eff }}$ mentioned above are only for Mira variables. A similar 


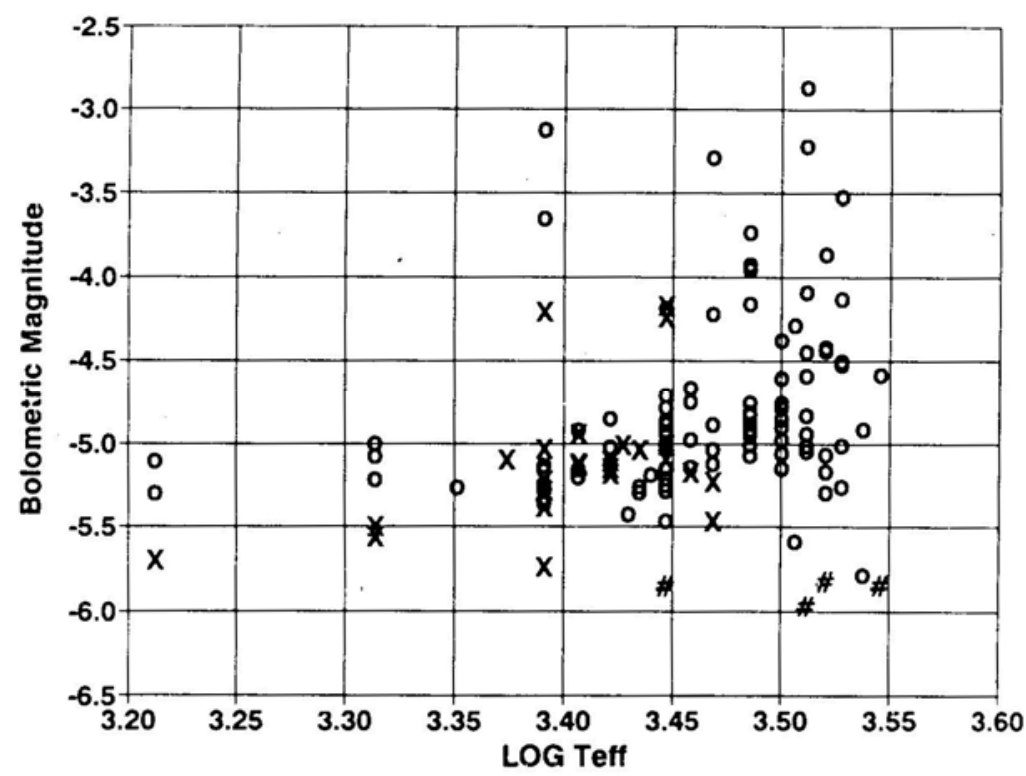

Figure 7. $\mathrm{H}-\mathrm{R}$ diagram for the observed masing and non-masing sources. ' $\mathrm{X}$ ' $=$ maser, 'o' = non-maser and ' $\#$ ' = supergiant star.

conclusion for supergiants or variables of different types can be made only after more such stars are observed.

The limiting values of $M_{\mathrm{bol}}$ and $T_{\text {eff }}$ may be indicating that a Mira variable has to attain a certain age before the onset of the $\mathrm{SiO}$ maser emission. On the other hand, using

$$
M_{\text {bol }}=42.31-5 \log \left(\frac{R}{R_{\odot}}\right)-10 \log T_{\text {eff }},
$$

the limiting values of $M_{\text {bol }}$ and $T_{\text {eff }}$ imply a lower limit on the stellar radius of $\approx 300 \mathrm{R}_{\odot}$, below which the $\mathrm{SiO}$ maser emission is suppressed. A large value of radius may simply allow a larger path length for the gain of the maser, and hence a greater maser luminosity. It also implies the density of gas in the envelope to be low, a condition which is favourable for non-LTE.

\subsection{Correlation of Maser Luminosity with Spectral Type and Bolometric Magnitude}

In Figs. 8 and 9, we have plotted the maser photon luminosity, and the maser flux as a function of distance. These figures show that the maser sources are not equally luminous, and the variation in the luminosity is intrinsic to the source. Fig. 10 shows the $\mathrm{SiO}$ maser photon luminosity as a function of the mean spectral type (MST). The intrinsic properties of super-giants represented by '\#' are expected to be different from those of the regular M-giant Mira variables. But for the three super-giants, all the masing Mira variables have MST later than M6. As a Mira variable approaches the maximum visual brightness during its pulsation (i.e. the zero phase), its spectral class 


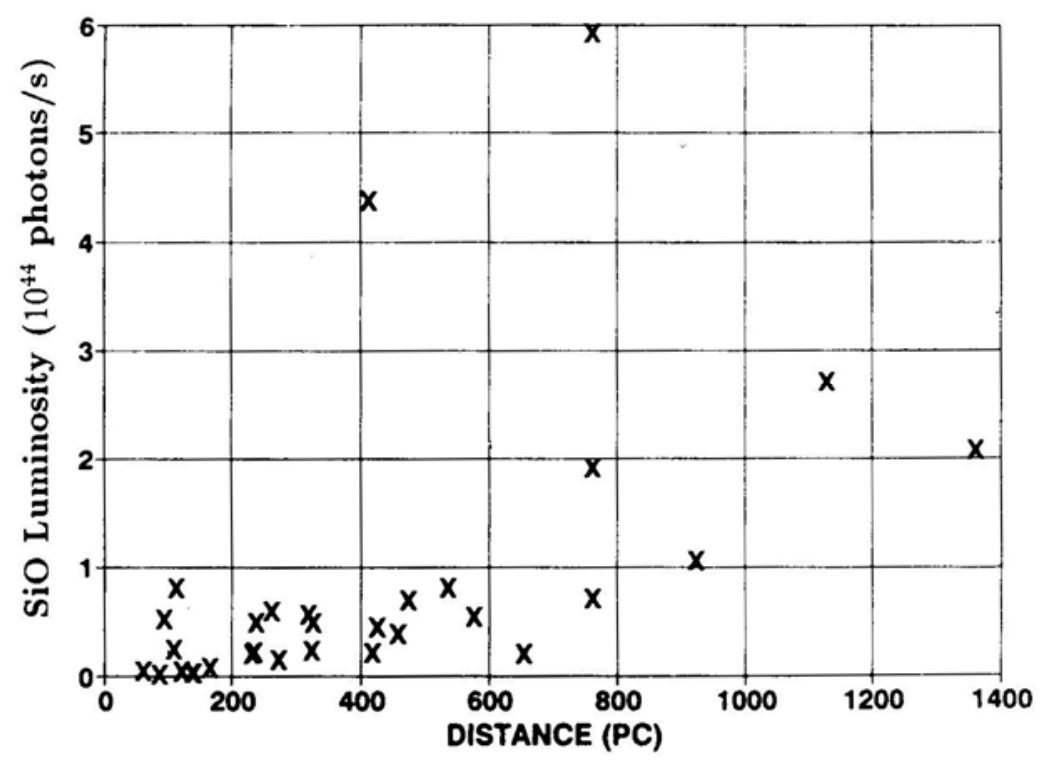

Figure 8. The SiO luminosity of maser sources versus their distances.

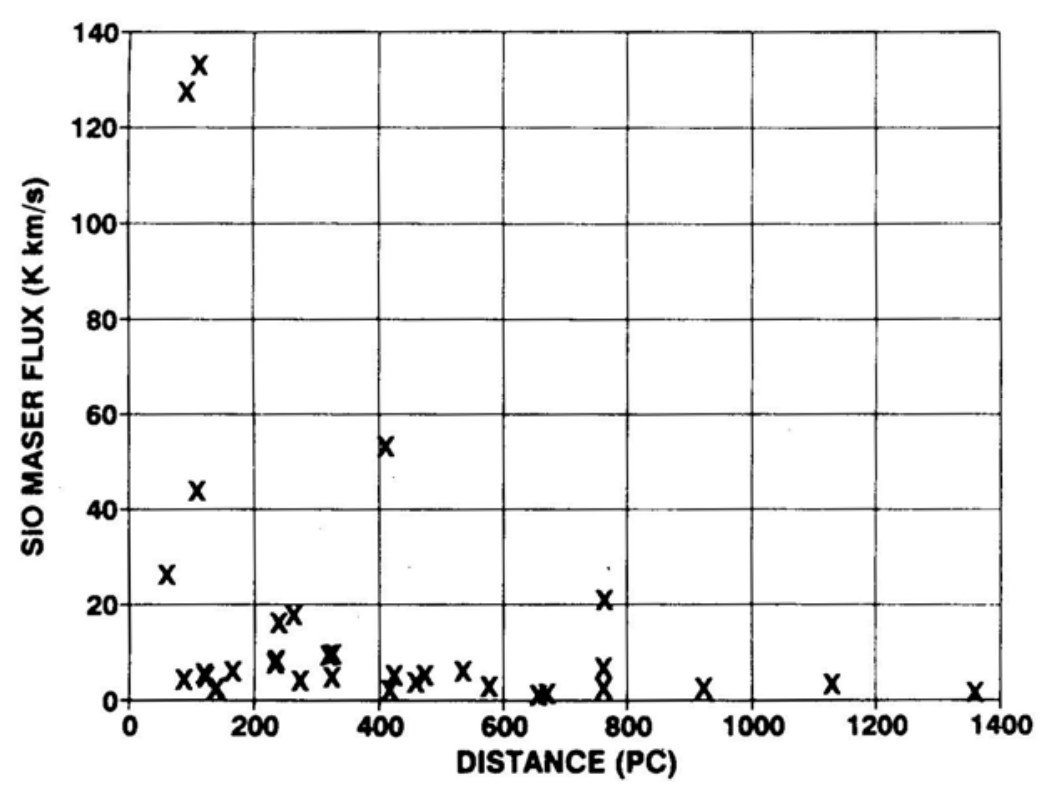

Figure 9. The $\mathrm{SiO}$ flux of maser sources versus their distances.

approaches an earlier type (corresponding to a hotter temperature). The limiting spectral type below which there is no maser emission is therefore expected to be even earlier in terms of the zero phase spectral type (ZPST) than the MST. In a plot similar to Fig. 10, instead of MST we used ZPST which was available for some sources from Keenan, Garrison \& Deutsch (1974). The limiting value in terms of ZPST was found 


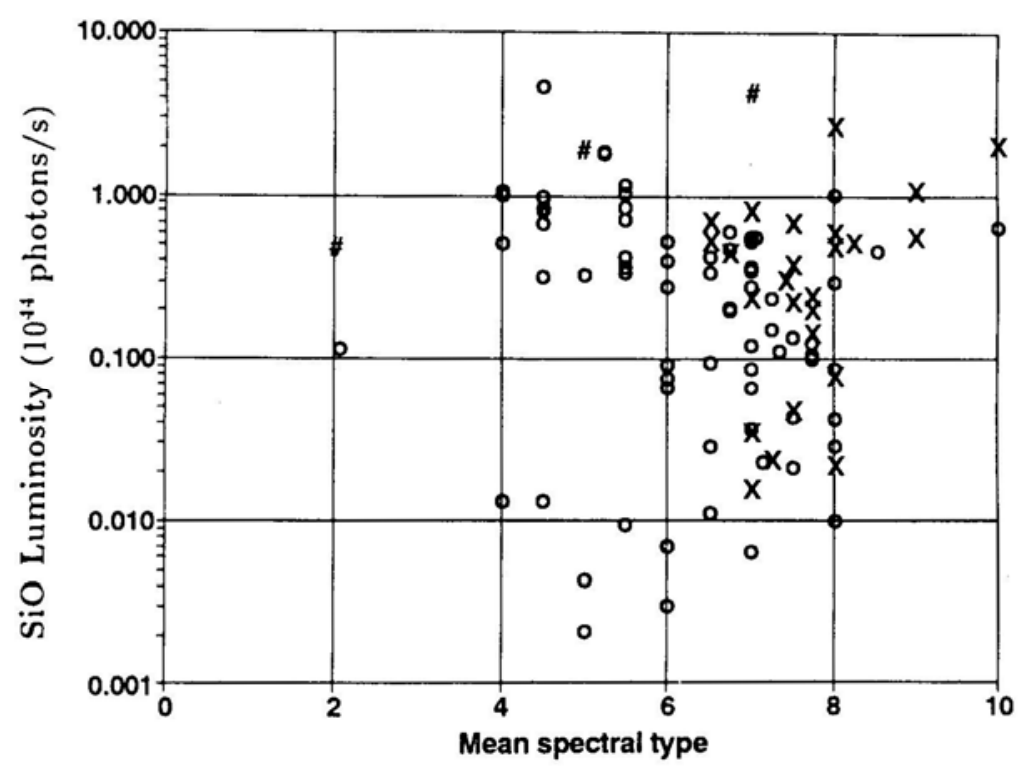

Figure 10. A plot of the SIO maser luminosity versus the mean spectral-type of the star. ' $\mathrm{X}$ ' = maser, 'o' = non-maser and '\#' = supergiant star.

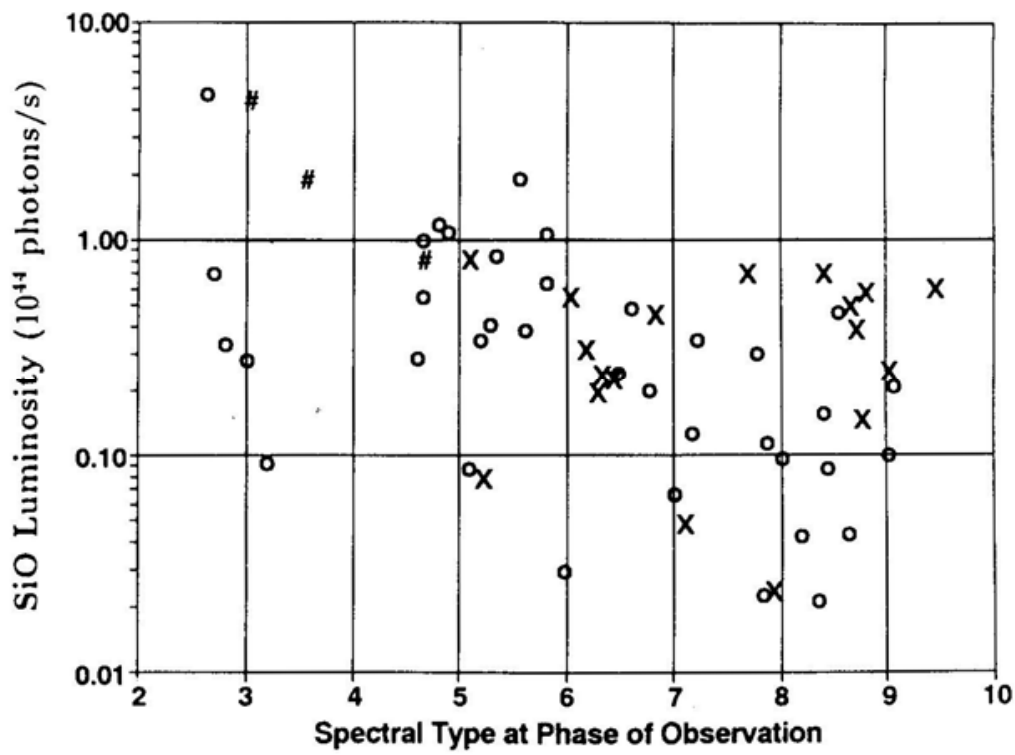

Figure 11. Same as Fig. 10 except the stellar spectral-type is the pulsation phase at he epoch of observation.

to be M5. Some of the sources for which we could find both, the distance as well as the phase of pulsation at the time of observation, are plotted in Fig. 11. Although this plot has a much smaller number of sources, we see that the masing Miras do not show a preferred value of spectral type in the range M6 -M10. The range of spectral types for which the maser emission occurs is thus not smaller than that for the MST. 
There are a substantial number of non-masers even in the spectral range M6-M10, as listed in Table 2. To check if they are at phases which correspond to the minimum $\mathrm{SiO}$ luminosity, we have plotted their distribution with phase (see Fig. 12). Although the number of sources in this histogram is rather small, there is no indication of a preferred phase implying that being at the phase of minimum $\mathrm{SiO}$ emission cannot

Table 2: Non-detections among Mira variables with mean spectral-type > M6.

\begin{tabular}{|c|c|c|c|c|c|c|c|c|c|}
\hline No. & Source & $\begin{array}{c}\text { Variable } \\
\text { type }\end{array}$ & $\begin{array}{c}\text { Spectral } \\
\text { type }\end{array}$ & $M_{\text {bol }}$ & $\begin{array}{c}\Delta m_{v} \\
\text { AAVSO }\end{array}$ & $\begin{array}{c}\Delta m_{\mathrm{v}} \\
\text { GCVS }\end{array}$ & $\begin{array}{l}\text { Prev. } \\
\text { Det. }\end{array}$ & $\begin{array}{c}\mathrm{SiO} \text { lum. } \\
\left(\times 10^{44}\right)\end{array}$ & Phase \\
\hline 1 & Ant $\mathrm{V}$ & M & M7IIIE & -4.91 & & & $a, c$ & & 0.46 \\
\hline 2 & Aql RT & M & $\mathrm{M} 6 \mathrm{E}-\mathrm{M} 8 \mathrm{E}(\mathrm{S})$ & -5.00 & 5.6 & 6.9 & $a, c$ & 0.07 & 0.95 \\
\hline 3 & Ari U & M & M4E-M9.5E & -5.13 & & 8.0 & $a, c$ & 0.21 & 0.74 \\
\hline 4 & Aur R & M & M6.5E-M9.5E & -5.35 & 7.0 & 7.2 & $a, c$ & 0.03 & 0.11 \\
\hline 5 & Aur U & M & M7E-M9E & -5.23 & 5.5 & 8.0 & $\mathrm{a}, \mathrm{c}$ & 0.09 & 0.84 \\
\hline 6 & Cam W & M & M7 & -4.85 & & 3.7 & & & 0.43 \\
\hline 7 & Cnc W & M & M6.5E-M9E & -5.19 & & 7.0 & $\mathbf{a}, \mathbf{c}$ & 0.13 & 0.33 \\
\hline 8 & CvN T & M & M6.5E & -4.87 & & 5.0 & & 0.45 & \\
\hline 9 & $\mathrm{CMa} \mathrm{DN}$ & M & M8 & & & & & & $\cdot$ \\
\hline 10 & CMi S & M & M6E-M8E & -5.01 & & 6.6 & $\mathrm{a}, \mathrm{c}$ & 0.13 & \\
\hline 11 & Cas $\mathrm{Y}$ & M & M6E-M8.5E & -5.24 & & 6.6 & $a, b$ & 0.16 & 0.68 \\
\hline 12 & Cen RT & M & M6-M7.5 & -4.73 & & 5.5 & & 0.50 & 0.40 \\
\hline 13 & Cen UU & M & M8E.5 & -5.12 & & 4.0 & & & 0.63 \\
\hline 14 & Cep T & M & M5.5E-M8.8E & -5.18 & 4.3 & 6.1 & $a, b$ & 0.02 & 0.85 \\
\hline 15 & Com R & M & M5E-M8EP & -5.11 & 5.7 & 7.5 & $a, b, c$ & 0.35 & 0.23 \\
\hline 16 & CrV R & M & M4.5E-M9E & -4.96 & 6.3 & 7.7 & & 0.21 & 0.95 \\
\hline 17 & Cyg Z & M & M5E-M9E & -4.77 & 4.6 & 7.6 & $\mathbf{a}, \mathbf{c}$ & 0.29 & 0.15 \\
\hline 18 & Cyg SX & M & $\mathrm{M} 7 \mathrm{E}$ & -5.24 & & 7.0 & & 0.56 & \\
\hline 19 & Dra $R$ & M & M5E-M9EIII & -4.69 & & 6.5 & & 0.37 & \\
\hline 20 & Eri W & M & M7E-M9 & -5.15 & 5.2 & 7.0 & $\mathrm{a}, \mathrm{c}$ & 0.30 & 0.39 \\
\hline 21 & Gem UZ & M & M9 & -5.06 & & & & & 0.89 \\
\hline 22 & Hya X & M & M7E-M8.5E & -4.91 & & 6.4 & $\mathrm{a}, \mathrm{c}$ & 0.11 & \\
\hline 23 & Lep T & $\mathrm{M}$ & $\mathrm{M} 6 \mathrm{E}-\mathrm{M} 9 \mathrm{E}$ & -5.12 & 4.6 & 6.9 & $\mathrm{a}, \mathrm{c}$ & 0.04 & 0.64 \\
\hline 24 & Lep RT & M & M9E & -5.21 & & & & & 0.55 \\
\hline 25 & Lib FS & M & M8.1-M9 & -5.25 & & & & 0.48 & 0.41 \\
\hline 26 & Lup R & M & M5.5-M8 & -4.65 & 4.0 & 4.6 & & 0.65 & 0.26 \\
\hline 27 & Lyr V & M & $\mathrm{M} 7 \mathrm{E}$ & -5.13 & 5.1 & 7.5 & & 0.58 & 0.55 \\
\hline 28 & Lyr RW & M & $\mathrm{M} 7 \mathrm{E}$ & -5.45 & 4.3 & 6.2 & b & 0.38 & 0.67 \\
\hline 29 & Mon ER & M & M9: & -4.99 & & & & & 0.13 \\
\hline 30 & Mon GN & M & M9: & & & & & & \\
\hline 31 & Oph RT & $\mathrm{M}$ & $\mathrm{M} 7 \mathrm{E}(\mathrm{C})$ & -5.27 & 5.5 & 6.9 & $a, b$ & 0.59 & \\
\hline 32 & Oph V1111 & M & M4III-M9 & & & 4.0 & & & \\
\hline 33 & Ori S & M & M6.5E-M9.5E & -5.24 & & 6.8 & $a, b$ & 0.04 & 0.92 \\
\hline 34 & Ori EP & M & M10E & -5.09 & & 2.5 & & & 0.28 \\
\hline 35 & Pic S & M & M6.5E-M8III-IIE & -5.28 & 5.7 & 7.5 & & 0.25 & 0.19 \\
\hline 36 & Sgr RR & M & $\mathrm{M} 4 \mathrm{E}-\mathrm{M} 9 \mathrm{E}$ & -5.02 & 6.4 & 8.6 & $\mathrm{a}, \mathrm{c}$ & 0.10 & 0.60 \\
\hline 37 & Sco RR & M & M6II-IIIE-M9 & -4.84 & 5.9 & 7.4 & b & 0.02 & 0.59 \\
\hline 38 & Tau R & M & M5E-M9E & -4.98 & 5.6 & 8.2 & $\mathrm{a}, \mathrm{c}$ & 0.09 & 0.21 \\
\hline 39 & Umi S & M & M6E-M9E & -5.01 & & $>5.7$ & $\mathrm{a}, \mathrm{c}$ & 0.14 & \\
\hline
\end{tabular}

Notes: a: Previous detection at $43 \mathrm{GHz}$.

b: Previous detection at $86 \mathrm{GHz}$.

c: Previous non-detection at $86 \mathrm{GHz}$.

*: Benson et al. (1990). 


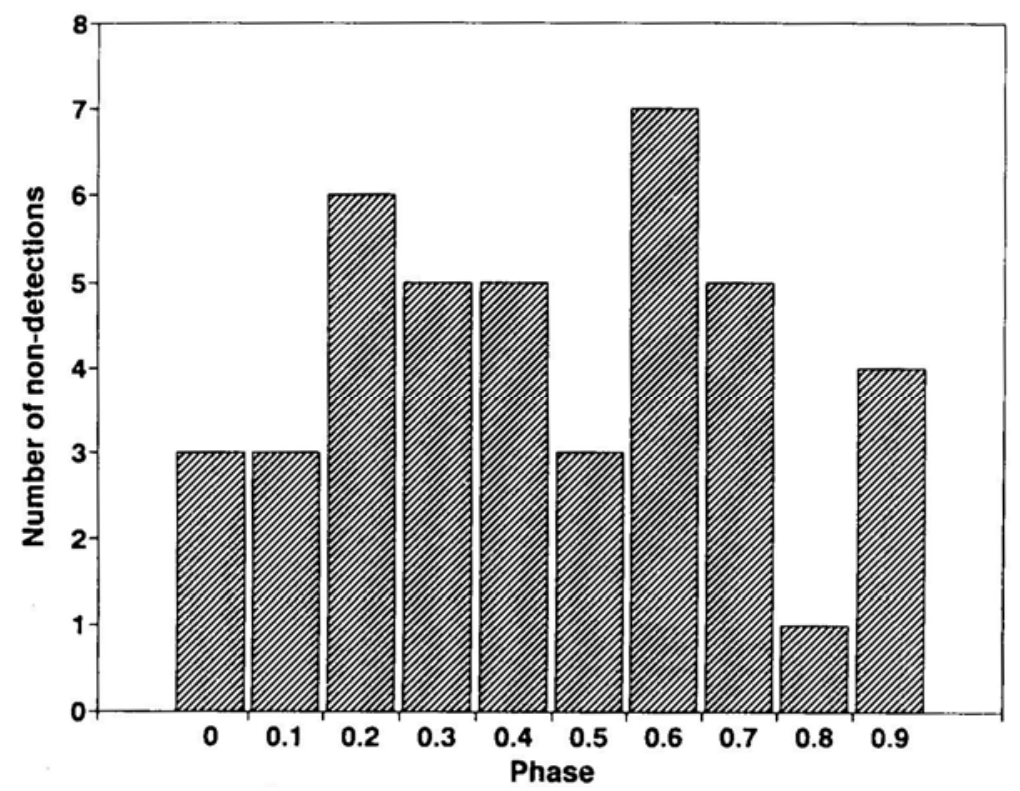

Figure 12. Distribution in the pulsation phase of Mira variables in which $\mathrm{SiO}$ maser emission was not detected.

be the sole cause behind their non-detection. Other possibilities are discussed in section 5 .

Thus, spectral type later than M6 is a necessary but not a sufficient condition for masing. Fig. 13 shows that the masing Miras are brighter than -4.2 in bolometric magnitude. Thus, another limiting factor is the bolometric magnitude. In fact, many of the non-masers in the spectral type range M6-M10 have bolometric magnitudes fainter than $-4.8 \mathrm{~m}$. Fig. 13 also shows a tendency for the maser luminosity to decrease with increasing bolometric magnitude; this is discussed in Patel \& Shukre (1992).

\subsection{Dependence of Maser Luminosity on the Amplitude of Pulsation}

Another possible intrinsic property of Mira variables to which the $\mathrm{SiO}$ maser may be related, is the amplitude of pulsation. Mira variables show very large changes in the visual magnitude. The amplitudes of pulsation at infrared wavelengths on the other hand, are much smaller (about 1 magnitude at infrared, for an amplitude of about 5 magnitude at visual). This is expected due to variation in the temperature between $2000 \mathrm{~K}$ to $2500 \mathrm{~K}$ during pulsation, for a typical Mira variable. Apart from changes in temperature, the amplitude of pulsation will reflect changes in the diameter of the star; and since the $\mathrm{SiO}$ maser exists very close to the photosphere, we may expect a physical connection between the maser process and the amplitude of pulsation. To investigate this relation, we have plotted the $\mathrm{SiO}$ maser photon luminosity versus the pulsational visual amplitude $\Delta m_{v}$. Fig. 14 shows a tentative anti-correlation between the maser luminosity and $\Delta m_{v}$ obtained from GCVS. In 


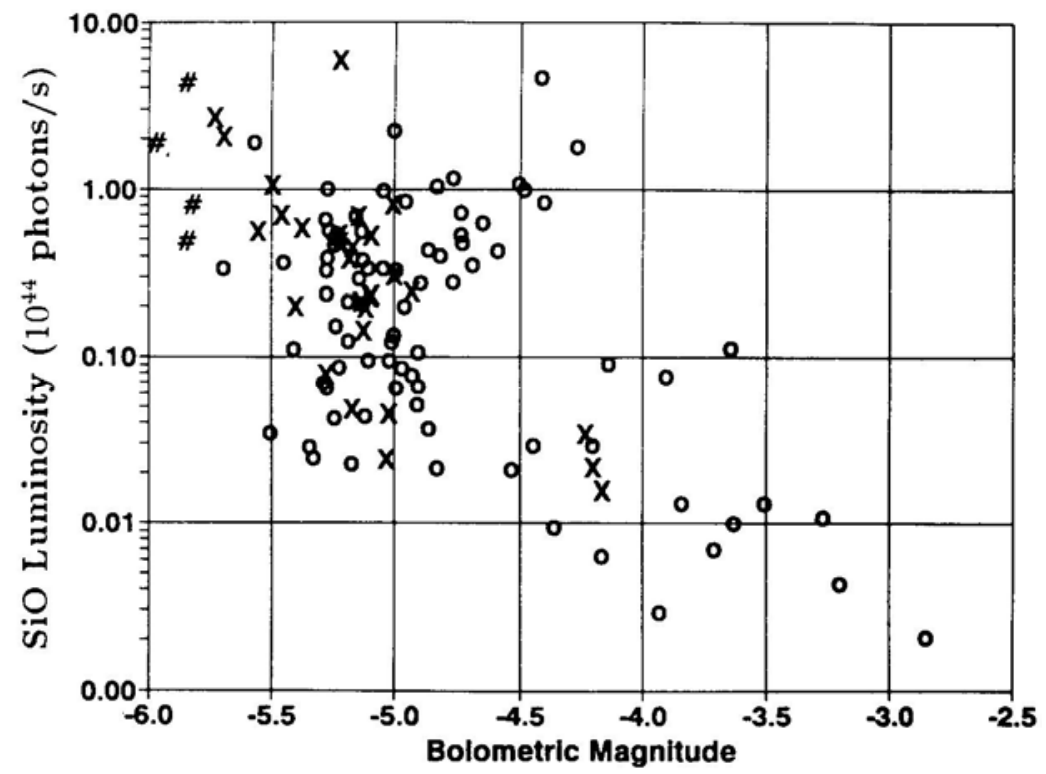

Figure 13. A plot of $\mathrm{SiO}$ maser luminosity versus the bolometric magnitude of the source, ' $\mathrm{X}$ ' = maser, ' $\mathrm{o}$ ' = non-maser and '\#' = supergiant star.

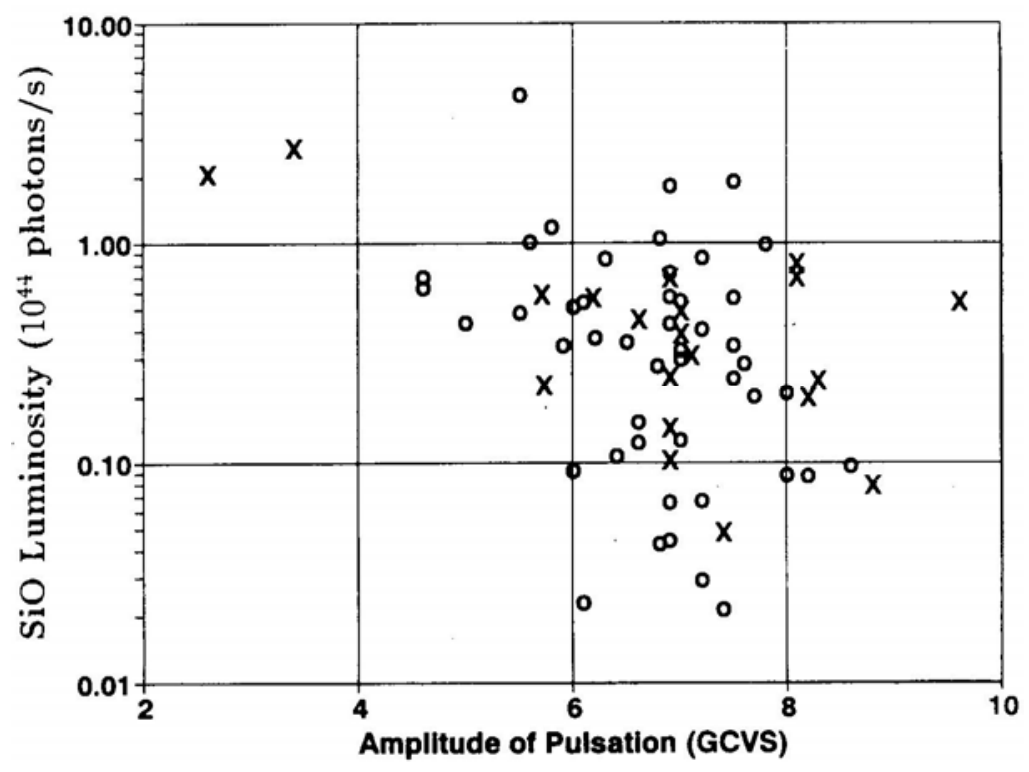

Figure 14. A plot of $\mathrm{SiO}$ maser luminosity of Mira variables versus their visual amplitude of the pulsation. The amplitude in magnitude units are from GCVS (Kholopov 1985. ' $\mathrm{X}$ ' = maser and 'o' non-maser.

GCVS (Kholopov 1985) a value of $\Delta m_{v}$ is available for almost all the sources in our list. These are recent values of $\Delta m_{v}$ where as the AAVSO $\Delta m_{v}$ (and the 'notes' in the third edition of GCVS) represent mean values which were not available for all the sources in Fig. 14. 
This result needs to be confirmed by observing more sources and using the mean values of $\Delta m_{v}$. It may also be useful to check for this anti-correlation by plotting the ratio of $\mathrm{SiO}$ maser and thermal $(v=0)$ emission against $\Delta m_{v}$. We expect such a comparison to be free of distance uncertainties and independent of $\mathrm{SiO}$ abundance variation from source to source. Further, the visual amplitude has severe molecular blanketing effects (Lockwood \& Wing 1971, Whitelock 1990); hence it is perhaps more reliable to use infrared amplitude where molecular absorption is minimal.

It is important to note that the correlations we have discussed are independent of each other. The bolometric magnitude, spectral type and amplitude of pulsation for stars in our sample, do not show any correlation with each other.

\section{Interpretations}

\subsection{Non-detections}

There are a large number of Mira variables with no detected $\mathrm{SiO}$ maser emission. In Table 2, we see that there are several Mira variables in the range of spectral types M6-M10, for which we only have upper-limits on the SiO maser luminosity. The limits for several of these are much below the luminosities for the masing sources detected in our sample. This raises the question - Why are these Mira variables such weak masers if they are masing at all? In Table 2, we find that there are many sources which have been detected previously, at 43 and $86 \mathrm{GHz}$. These sources are marked by ' $a$ ' and ' $b$ ' respectively, in column 7. Generally, the $43 \mathrm{GHz}$ emission is expected to be about twice as strong as the $86 \mathrm{GHz}$ (Snyder \& Buhl 1975). Therefore, those sources which are already weak at $43 \mathrm{GHz}$ should be even weaker at $86 \mathrm{GHz}$ and thus will not be detectable with our sensitivity. On the other hand, there are many sources previously detected at $86 \mathrm{GHz}$, which we have failed to detect. We look at these and other non-detections in the light of the dependence of the maser power on intrinsic stellar properties discussed above.

For those sources for which data is insufficient, obviously, no statement can be made. Of the remaining, those which have $M_{\mathrm{bol}}>-4.8$ are expected to be weak masers.

The dependence on the phase of pulsation $\phi$ affects the maser emission in two ways. Because the spectral type varies with $\phi$, for some stars (e.g. R Com, R Tau) the spectral type at the phase of our observation would have been earlier than M6, suppressing the maser emission. Secondly, the maser output is expected to vary with $\phi$ such that it will be low in the range $0.5<\phi<0.9$. This of course will be of more significance for those sources whose stellar parameters have values close to the cut-offs or are at a large distance (e.g., T Lep, RW Lyr).

The coordinates used for our observation of $\mathrm{Y}$ Cas were not precise. For some other sources (e.g., TCep, UUCen), the data is noisier than average $(\sigma \cong 0.10 \mathrm{~K})$. In a few cases, just the large distance could push the flux level below our sensitivity limit, e.g., comparing W Cnc and R Hya we see that the flux for the former would be $\approx 1 / 30$ of that for the latter.

The non-detection of the maser emission from sources in Table 2 can thus be understood in terms of the factors discussed above. It lends indirect support to the conclusions of previous sections. Only two sources (RT Aql and S Ori) cannot be 
understood in these terms. It should be noted that at least a few of the sources are expected to show maser emission if observations are made with better sensitivity (e.g. W Cnc, W Eri) or at a proper phase (e.g. S CMi, X Hya).

\subsection{Dependence of Maser Power on Spectral Type}

The $\mathrm{SiO}$ maser power clearly must depend on the molecular abundance of $\mathrm{SiO}$ in the atmosphere of the Mira variable. As seen earlier, one of the main results that has emerged from our observations is that the masing Mira variables have spectral types later than M6. Indeed, this dependence of the maser power on the spectral type could simply be due to a lower abundance of $\mathrm{SiO}$ in stars having spectral types earlier than M6. Such an abundance variation was in fact predicted by Johnson, Beebe \& Sneden (1975). Rinsland \& Wing (1982) ${ }^{1}$ observed the vibrational-rotational transitions in $\mathrm{SiO}$, at $4 \mu \mathrm{m}$ from several M-giants and a few Mira variables of various spectral types. They found that the equivalent width of the absorption band increased with the spectral type, showing that above a temperature of $\sim 3000 \mathrm{~K}$, there was a steep decline in the value of the equivalent width which could not be due to the dissociation of SiO. A similar effect is also expected for the abundance of water (Tsuji 1978). We therefore suggest that a reduction in the abundance of $\mathrm{SiO}$ in earlier spectral types is the cause for the observed dependence of the $\mathrm{SiO}$ maser power on effective temperature (spectral type). This can be tested by future observations of $\mathrm{SiO}$ in thermal rotational transitions from Mira variables covering a wide range of spectral types.

\section{Conclusions}

There seem to be two criteria which may inhibit a Mira variable from giving rise to $\mathrm{SiO}$ maser emission at $86 \mathrm{GHz}$ : They are: (1) Spectral type earlier than M6 (or $\log T_{\text {eff }} \geqslant 3.48$ ) and (2) Bolometric magnitude fainter than -4.8 magnitudes.

\section{Acknowledgement}

This work would not have been possible without the continuous effort and enthusiasm of everyone at the millimeter-wave laboratory, electronics laboratory and the workshop of the Raman Research Institute. We are particularly grateful to P. G. Ananthasubramanian, K. Sukumaran, G. Rengarajan, R. Nandakumar, M. Selvamani, K. Chandrashekara and the telescope operators for their help in carrying out the observations; and to V. Radhakrishnan, N. V. G. Sarma, C. S. Shukre and R. Nityananda for their guidance and many helpful discussions. We also thank K. R. Anantharamaiah for helpful comments on the manuscript. It is a pleasure to thank Janet Mattei and the AAVSO for providing us the visual light-curves of most of the Mira variables in our sample. We are grateful to the referee for several useful comments.

\footnotetext{
${ }^{1}$ We are grateful to Dr. T. Tsuji for this reference.
} 


\section{References}

Alcock, C, Ross, R. R. 1986, Astrophys. J., 310, 838.

Alcolea, J., Bujarrabal, V., Gallego, J. D. 1989, Astr. Astrophys., 211, 187.

Benson, P. J., Little-Marenin, I. R., Woods, T. C, Attridge, J. M., Blaise, K. A., Rudolf, D. B., Rubiera, M. E., Keefe, H. L. 1990, Astrophys. J. Suppl., 74, 911.

Bessell, M. S., Brett, J. M., Scholz, M., Wood, P. R. 1989, Astr. Astrophys., 213, 209.

Cahn, J. 1977, Astrophys. J. Lett., 212, L135.

Catchpole, R. M., Robertson, B. S. C, Lloyd-Evans, T., Feast, M. W. 1979, South African Astronomical Observatory Circulars, 1, 61.

Dickinson, D. F., Snyder, L. E., Brown, L. W., Buhl, D. 1978, Astr. J., 83, 36.

Dyck, H. M, Lockwood, G. W., Capps, R. W. 1974, Astrophys. J., 189, 89.

Engels, D. 1979, Astr. Astrophys. Suppl. Ser., 36, 337.

Engels, D., Heske, A. 1989, Astr. Astrophys. Suppl. Ser., 81, 323.

Feast, M. W., Robertson, B. S. C, Catchpole, R., Lloyd-Evans, T., Glass, I. S., Carter, B. S. 1982, Mon. Not. R. astr. Soc., 201, 439.

Feast, M. W. 1984, Mon. Not. R. astr. Soc., 211, 51.

Gezari, D. Y., Schmitz, M., Mead, J. M. 1984, Catalog of Infrared Observations, NASA Ref. Publ. 1118.

Ikaunieks, J. 1975, in Pulsating Stars Ed. B. V. Kukarkin, John Wiley and Sons. p. 259.

Jewell, P. R., Walmsley, C. M., Wilson, T. L., Snyder L. E. 1985, Astrophys. J., 298, L55.

Johnson, H. R., Beebe R. F., Sneden, C. 1975, Astrophys. J. Suppl. Ser., 29, 123.

Keeley, D. A. 1970, Astrophys. J., 161, 643.

Keenan, P. C., Garrison, R. F., Deutsch, A. J. 1974, Astrophys. J. Suppl. Ser., 28, 271.

Kholopov, P. N. (Ed), 1985, General Catalogue of Variable Stars, Moscow Publishing House.

Lane, A. P. 1984, in IAU Symp. 110, VLBI and compact radio sources, Eds R. Fanti, K. Kellerman, \& G. Setti, Reidel Publ. 1984 p. 329.

Lloyd Evans, T. 1990, Mon. Not. R. astr. Soc., 243, 336.

Lockwood G. R., Wing R. F. 1971, Ap. J., 169, 63.

Lockwood, G. R. 1972, Ap. J. Suppl. Ser., 24, 373.

McIntosh, G. C, Predmore, C. R., Moran, J. M., Greenhill, L. J., Rogers, A. E. E., Barvainis, R. 1989, Ap. J., 337, 934.

Moran, J. M., Ball, J. A., Predmore, C. R., Lane, A. P., Reid, M. J., Hansen, S. S. 1979, Ap. J., 231, L 67.

Patel, N. A. 1990, Ph.D. Thesis, Indian Institute of Science, Bangalore.

Patel, N. A., Shukre C. S. 1992, submitted to Astrophys. J.

Rinsland, C. P., Wing, R. F. 1982, Ap. J., 262, 201.

Robertson, B. S. C, Feast, M. W. 1981, Mon. Not. R. astr. Soc., 196, 111.

Sahai, R. 1987, in I. A. U. Symposium 120. Astrochemistry, Eds M. S. Vardya \& S. P. Tarafdar, D. Reidel Pub. Co., p 341.

Scalo, J. M. 1976, Ap. J., 206, 474.

Snyder, L. E., Buhl, D. 1975, Ap. J., 197, 329.

Spencer, J. H., Waak, J. A., Bologna, J. M., Schwarz, P. R. 1977, Astron. J., 82, 706.

Tsuji, T. 1978, Astron. Astrophys., 62, 29.

Tsuji, T. 1981, Astron. Astrophys., 99, 48.

Vardya, M. S., 1989, in Evolution of Peculiar Red Giants, Eds H. R. Johnson, B. Zuckermann, Cambridge: Cambridge University Press, p. 349.

Whitelock, P. A. 1990 in Confrontation between stellar pulsation and evolution, EdC. Cacciari, Astron. Soc. Pacific Conference Series, to be published. 